PHYSICAL REVIEW D 92, 063525 (2015)

\title{
Constraining ultralarge-scale cosmology with multiple tracers in optical and radio surveys
}

\author{
D. Alonso and P. G. Ferreira \\ University of Oxford, Denys Wilkinson Building, Keble Road, Oxford, OX1 3RH, United Kingdom
}

(Received 14 July 2015; published 22 September 2015)

\begin{abstract}
Multiple tracers of the cosmic density field, with different bias, number and luminosity evolution, can be used to measure the large-scale properties of the Universe. We show how an optimal combination of tracers can be used to detect general-relativistic effects in the observed density of sources. We forecast for the detectability of these effects, as well as measurements of primordial non-Gaussianity and large-scale lensing magnification with current and upcoming large-scale structure experiments. In particular we quantify the significance of these detections in the short term with experiments such as the Dark Energy Survey (DES), and in the long term with the Large Synoptic Survey Telescope (LSST) and the Square Kilometre Array (SKA). We review the main observational challenges that must be overcome to carry out these measurements.
\end{abstract}

DOI: $10.1103 /$ PhysRevD.92.063525

PACS numbers: 98.80.-k, 98.80.Es, 98.80.Jk

\section{INTRODUCTION}

Over the next few decades we expect to map out the large scale structure of the observable Universe in its entirety. By this, we mean that we should be able to completely characterize the spatial distribution of matter from subMegaparsec scales out to the cosmological horizon, from the present epoch out to the highest observable redshift, deep into the dark ages of structure formation. We will achieve this through a combination of redshift (both spectroscopic and photometric) and weak lensing surveys allied with intensity mapping and cosmic microwave background (CMB) experiments. From the spatial distribution of matter, we should be able to infer the structure of space-time within our cosmic horizon and in doing so dramatically improve our understanding of the cosmological model and its fundamental assumptions.

Key to our understanding of the Universe are the very largest scales. There we expect to be truly sensitive to primordial and general-relativistic aspects of cosmology and, one hopes, we should be able to constrain aspects of the Universe which are not accessible if we restrict ourselves to smaller, Newtonian, scales. The most notable aspects, which we will focus on in this paper and on which we will elaborate below, are general-relativistic effects on number counts and luminosities [1-5], large-scale weak lensing and the scale dependence of bias due to primordial non-Gaussianity [6,7].

There have been attempts at constraining the largest scales of the Universe with current surveys [8] and of quantifying how well we will be able to do so with future experiments [9-14]. Most of these attempts have focused on autocorrelations of single tracers, where it has become clear that cosmic (or sample) variance is sufficiently large that the resulting constraints are not (or will not be) particularly stringent. In other words, the fundamentally stochastic nature of the seeds for structure combined with the finite volume of any survey severely restricts the number of independent modes we can sample and introduces a large and inescapable floor on the uncertainty of any of the fundamental quantities we wish to constrain. In [15] we undertook a detailed, comprehensive analysis of the four different types of surveys of large scale structure which are currently being planned, finding indeed that there were severe limitations to how well we could constrain large scale effects.

An ingenious and alternative approach can be taken if one has multiple tracers (such as galaxies or line-emitting species) with different bias and luminosity functions. As was first shown in [16] and then further developed in [17], with multiple tracers it is possible to effectively "divide out" the stochastic dependence of the cosmological density field allowing us to beat cosmic variance for a few, specific, observables such as the growth rate and clustering bias. While this approach was originally advocated to target the scale dependent bias of primordial non-Gaussianity [16], we expect that such an effect naturally extends to the general-relativistic corrections in large-scale structure surveys.

In this paper, we further flesh out the idea of using multitracer methods to constrain what we dub ultralargescale effects. We focus on surveys that have two key properties. First, they must be able to cover large volumes of space with an appreciable number density of sources. Second, we need to be able to pick out two or more different tracers with a sufficiently high bias but also with sufficiently different evolution properties that the multitracer technique can be effective. In subsection III B we derive a simple expression which can be used as a guide for what the best combinations of tracers (and their properties) are to efficiently constrain general relativistic effects. 


\section{ALONSO AND P. G. FERREIRA}

Both of these requirements lead us to choose photometric redshift surveys [focusing on Dark Energy Survey (DES) and Large Synoptic Survey Telescope (LSST)], radiocontinuum surveys and intensity mapping surveys [primarily from the Square Kilometre Array (SKA)]. Indeed we find that a combination of these surveys will give us remarkable constraints on ultralarge-scale effects.

We structure our paper as follows. In Sec. II we remind ourselves of the various effects that come into play on large scales, explaining their origin and how they affect the observables we will consider. In Sec. III we describe the Fisher forecasting formalism we will be using and employ it to derive a simple expression which shows how the multitracer method works to our advantage, for isolating the general-relativistic corrections to large-scale surveys. This expression gives us a few pointers to what the optimal surveys will be for us to achieve our goals. In Sec. IV we focus on optical and near infrared photometric redshift surveys, specifically on LSST, in Sec. V we look at cosmological radio surveys, specifically continuum and intensity mapping as will be carried out by the SKA, and in Sec. VI we will see how cross-correlating these different surveys will contribute to our overall goals. Our results are truly promising and in Sec. VII their limitations and future prospects are presented. In particular we speculate on how precise measurements on ultralarge scales will contribute to our understanding of the fundamental cosmological model as well as how they might help us further characterize the large scale properties of the Universe.

\section{COSMOLOGICAL OBSERVABLES ON ULTRALARGE SCALES}

The aim of this section is to introduce the two main observables on ultralarge scales that we will study here, as well as to establish the notation that will be used in the rest of the paper, which follows the one used in [15]. More thorough discussions of these topics can be found in, e.g. [5] for relativistic effects and [7] for primordial nonGaussianity.

\section{A. Relativistic effects in large-scale structure}

The aim of large-scale structure experiments is to map out the three-dimensional distribution of the matter density field in the Universe. This is most commonly done using the number counts of astrophysical sources in the sky, using their angular positions and redshifts as proxies for their three-dimensional coordinates. This means that we use the redshift and direction of the photons emitted by these sources in order to infer the comoving volume of the patch over which they are distributed; any perturbation in the trajectory or the frequency of these photons along the light cone, will affect our volume estimates, and consequently the measured three-dimensional density map.
Two perfect examples of such effects are redshift-space distortions (RSDs), by which the emitted photons are further redshifted by the peculiar velocities of the sources, and gravitational lensing magnification, the perturbation in the trajectory of the photons caused by the gravitational field of the intervening matter. RSDs displace the sources in redshift-space coherently along the line of sight, while lensing magnification modifies the solid angle subtended by a cluster of sources. While these two effects correspond to the two largest contributions to the total fluctuation in the observed number counts, after the intrinsic perturbation in the source number density, there are other nonzero contributions at linear order that potentially contain information about the nature of the gravitational interactions perturbing the photon trajectories. The complete set of terms at linear order was presented by [5] and [4], and can be classified into four main contributions:

$$
\Delta_{N}(z, \hat{\mathbf{n}})=\delta_{\mathcal{N}}+\frac{\partial \ln \overline{\mathcal{N}}}{\partial \eta} \delta \eta+\delta_{\|}+2 \delta_{\perp}\left[1-\frac{\partial \ln \overline{\mathcal{N}}}{\partial \ln L_{*}}\right],
$$

where $\overline{\mathcal{N}}\left(\eta, \ln L_{*}\right)$ is the physical number density of sources at conformal time $\eta$ with luminosities above $L_{*}$ (i.e. the cumulative luminosity function), related to the survey's flux limit $F_{\text {cut }}$ via

$$
L_{*}(z)=4 \pi(1+z)^{2} \chi^{2}(z) F_{\text {cut }} .
$$

The four terms on the right-hand side of Eq. (1) correspond to the intrinsic perturbation in the number density of sources $\left(\delta_{\mathcal{N}}\right)$, the difference between the source's conformal time $\eta$ and the conformal time inferred from the redshift $\eta_{*}(\delta \eta)$, and the corresponding differences in the radial distance $\left(\delta_{\|}\right)$and angular diameter distance $\left(\delta_{\perp}\right)$ of the sources. As discussed above, RSDs and lensing magnification contribute to $\delta_{\|}$and $\delta_{\perp}$, respectively. In a given gauge, we can expand each of these in terms of the metric, density and velocity perturbations. This has been done explicitly in the literature, and we present expressions for the complete set of terms in Appendix A.

Note that the amplitude of the observed perturbation in the number counts as a function of redshift depends on three tracer-specific quantities:

(i) The clustering bias $b$, relating the intrinsic perturbation in the number density with the fluctuations in the comoving-gauge matter perturbation, defined in Fourier space as $[5,18,19]$ :

$$
\delta_{\mathcal{N}}(k)=b \delta_{M}^{\mathrm{synch}}+\frac{\partial \ln \overline{\mathcal{N}}}{\partial \eta} \frac{v}{k},
$$

where $\delta_{M}^{\text {synch }}$ is the matter perturbation in the synchronous comoving gauge and $v$ is the peculiar velocity in the Newtonian gauge. 
(ii) The slope of the background number density of sources as a function of time, which is now customary to encode in the so-called evolution bias:

$$
f_{\mathrm{evo}} \equiv \frac{\partial \ln \left(a^{3} \overline{\mathcal{N}}\right)}{\partial \ln (a)}
$$

(iii) The slope of the number density of sources as a function of intrinsic luminosity, usually written in terms of the magnification bias:

$$
s \equiv \frac{2}{5} \frac{\partial \ln (\overline{\mathcal{N}})}{\partial \ln L_{*}} .
$$

In general, the astrophysical observable containing the most cosmological information is the perturbation in the number counts in a set of redshift bins

$$
\Delta_{i}(\hat{\mathbf{n}}) \equiv \int d z W_{i}(z) \Delta(z, \hat{\mathbf{n}})
$$

where $W_{i}(z)$ is the window function defining the $i$ th redshift bin (normalized to 1 when integrated over the whole redshift range). To make matters simpler, we will present our forecasts in terms of the harmonic coefficients of $\Delta_{i}(\hat{\mathbf{n}})$, defined as

$$
a_{\ell m}^{i} \equiv \int d \hat{\mathbf{n}} \Delta_{i}(\hat{\mathbf{n}}) Y_{\ell m}(\hat{\mathbf{n}}) .
$$

The main advantage of using the $a_{\ell m} \mathrm{~s}$ is that statistical isotropy makes them uncorrelated, so that:

$$
\left\langle a_{\ell m}^{i} a_{\ell^{\prime} m^{\prime}}^{j}\right\rangle \equiv C_{\ell}^{i j} \delta_{\ell \ell^{\prime}} \delta_{m m^{\prime}} .
$$

This equation also defines the angular power spectrum $C_{\ell}^{i j}$, containing all of the possible cross-correlations between two redshift bins $i$ and $j$.

As shown in [20], the angular power spectrum can be computed as

$$
C_{\ell}^{i j}=4 \pi \int_{0}^{\infty} \frac{d k}{k} \mathcal{P}(k) \Delta_{\ell}^{i}(k) \Delta_{\ell}^{i}(k),
$$

where $\mathcal{P}(k)$ is the dimensionless primordial power spectrum, which we assume to be close to scale invariant: $\mathcal{P}(k)=A_{s}\left(k / k_{0}\right)^{n_{s}-1}$, and $\Delta_{\ell}^{i}$ is a transfer function containing the terms in Eq. (1) projected along the radial direction in each redshift bin. In the Newtonian gauge $\Delta_{\ell}^{i}$ can be expanded as a sum of 10 individual terms,

$$
\begin{aligned}
\Delta \equiv & \Delta^{\mathrm{D}}+\Delta^{\mathrm{RSD}}+\Delta^{\mathrm{L}}+\Delta^{\mathrm{V} 1}+\Delta^{\mathrm{V} 2} \\
& +\Delta^{\mathrm{P} 1}+\Delta^{\mathrm{P} 2}+\Delta^{\mathrm{P} 3}+\Delta^{\mathrm{P} 4}+\Delta^{\mathrm{ISW}},
\end{aligned}
$$

where the complete expressions for each term are presented in Appendix A.
The first three terms, $\Delta^{\mathrm{D}}, \Delta^{\mathrm{RSD}}$ and $\Delta^{\mathrm{L}}$ are the dominant contributions to the total fluctuation [21], and correspond to the intrinsic perturbation in the source number density, the Kaiser-term [22] for redshift-space distortions and the effect of lensing magnification, respectively. The remaining 7 terms are subdominant and have never been accounted for, nor detected in the analysis of any data set. Of these, the terms $\Delta^{\mathrm{V} 1}$ and $\Delta^{\mathrm{V} 2}$ are extra RSD effects due to evaluating the background terms at the redshift perturbed by the Doppler effect, and the remaining terms correspond to similar effects caused by gravitational redshifting and lensing (e.g. the Shapiro time delay $\Delta^{\mathrm{P} 4}$ or the integrated Sachs-Wolfe effect $\left.\Delta^{\mathrm{ISW}}[23,24]\right)$. A great deal of the work presented in this paper is devoted to studying the detectability of these last 7 terms, and the potential benefits that such a detection could entail.

The contribution of these terms to the observed fluctuations is most relevant on the scale of the cosmic horizon, and even there they are subdominant with respect to the first three. Moreover, since accurate clustering measurements on these scales can be severely hampered by observational systematics, as we will review later, detecting the effect of these terms is very challenging. In particular, as was shown in [15], the amplitude of these terms is too small to be observable with any significance using any of the main cosmological probes targeted by next-generation largescale structure experiments individually. The main reason for this is the large cosmic variance on these scales, both due to the small number of superhorizon modes available at redshifts $z \lesssim 3$ and to the larger amplitude of matter fluctuations at late times. In order to detect these relativistic corrections, it is therefore necessary to reduce the statistical noise below the cosmic variance limit, a feat that might be achievable using the multitracer techniques that we review in Sec. III A. We will quantify the detectability of these general relativity (GR) corrections as was done in [15], by defining an effective parameter $\epsilon_{\mathrm{GR}}$ multiplying the terms $\Delta_{\ell}^{\mathrm{V} 1,2}, \Delta_{\ell}^{\mathrm{P} 1-4}$ and $\Delta_{\ell}^{\mathrm{ISW}}$. We do not include the lensing magnification term $\Delta_{\ell}^{\mathrm{L}}$ under the umbrella of $\epsilon_{\mathrm{GR}}$, since this effect has already been detected observationally $[25,26]$, and its contribution is also non-negligible on small angular scales. However, for completeness, and given that gravitational lensing is clearly a general-relativistic effect, we will also forecast for its detectability by defining a separate amplitude parameter for it, $\epsilon_{\mathrm{WL}}$.

To end this section, it is worth noting that, even though the form of the fluctuation $\Delta_{i}(\hat{\mathbf{n}})$ has been motivated for experiments targeting a flux-limited population of discrete sources, it is straightforward to extend it to the case of lineemission tomography [27] (most commonly called intensity mapping). The main difference between these two cases is that, while in a survey of discrete sources the proxy for the density perturbation is the fluctuation in the number of objects detected above a given flux in different patches of equal size, the observable in intensity mapping is the 
combined emission from all of the sources in these patches. Since lensing effects perturb angular separations in the same way as source luminosities, the net effect is an exact cancellation of the linear-order perturbation in the angular diameter distance for intensity mapping [(i.e. the $\delta_{\perp}$ term in Eq. (1)]. Thus, for the purposes of predicting the cosmological signal, intensity mapping experiments can be treated as a survey of discrete sources with a magnification bias given by the critical value $s_{\mathrm{IM}}=2 / 5$, and with an evolution bias given by:

$$
f_{\mathrm{evo}}^{\mathrm{IM}} \equiv \frac{\partial \ln \rho_{a}}{\partial \ln a},
$$

where $\rho_{a}$ is the background comoving density of the lineemitting species under study.

\section{B. Primordial non-Gaussianity}

One of the fundamental assumptions of the simplest versions of the standard cosmological model is an almostnegligible amount of non-Gaussianity in the statistics of the primordial density field [28]. A simple (although not universal) formalism [29] to quantify deviations with respect to this premise is to decompose the primordial gravitational potential $\Phi$ into a linear and a quadratic function of a Gaussian random field $\phi_{G}$ :

$$
\Phi=\phi_{G}+f_{\mathrm{NL}}\left(\phi_{G}^{2}-\left\langle\phi_{G}^{2}\right\rangle\right) .
$$

Since the matter density field gradually becomes nonGaussian due to the nonlinear nature of gravitational collapse, the level of primordial non-Gaussianity is easier to determine using early-Universe probes, such as the $\mathrm{CMB}$, which are well inside the linear regime. Current constraints based on higher-order CMB statistics favor a rather small level of non-Gaussianity, with $\left|f_{\mathrm{NL}}\right| \lesssim 7$ [30]. However, other statistical techniques have been developed with the aim of improving these constraints using lowredshift three-dimensional data sets. In particular it has been noted that a nonzero primordial non-Gaussianity would affect the formation and therefore the clustering statistics of biased tracers of the matter distribution [6,7]. The effect can be encoded as a modification of the Gaussian clustering bias $b_{G}(z) \rightarrow b_{G}(z)+\Delta b(z, k)$, with a scale dependence given by

$$
\Delta b(z, k)=3 f_{\mathrm{NL}} \frac{\left[b_{G}(z)-1\right] \Omega_{M} H_{0}^{2} \delta_{c}}{k^{2} T(k) D(z)},
$$

where $\delta_{c} \simeq 1.686$ is the linearized collapse threshold, $T(k)$ is the matter transfer function and $D(z)$ is the linear growth factor.

This scale-dependent bias induces extra power on large scales with a $\sim k^{-2}$ scale dependence, and it is easy to show that this extra power should be more evident on scales or the order of the horizon $k_{\mathrm{NG}} \sim f_{\mathrm{NL}} H_{0}$. Thus this signature of primordial non-Gaussianity presents characteristics that are very similar to the signal of the GR corrections presented in the previous sections (in fact it has been suggested that they could potentially be degenerate $[18,31,32])$, and therefore it makes sense to study both effects simultaneously.

Including our three main observables $\left(f_{\mathrm{NL}}, \epsilon_{\mathrm{GR}}\right.$ and $\left.\epsilon_{\mathrm{WL}}\right)$, the total number count fluctuation is

$$
\Delta_{\ell}=\Delta_{\ell}^{\mathrm{D}}\left(f_{\mathrm{NL}}\right)+\Delta_{\ell}^{\mathrm{RSD}}+\epsilon_{\mathrm{WL}} \Delta_{\ell}^{\mathrm{L}}+\epsilon_{\mathrm{GR}} \Delta_{\ell}^{\mathrm{GR}},
$$

where every term is implicitly scale and time-dependent, and $\Delta^{\mathrm{GR}} \equiv \Delta^{\mathrm{V} 1}+\Delta^{\mathrm{V} 2}+\Delta^{\mathrm{P} 1}+\Delta^{\mathrm{P} 2}+\Delta^{\mathrm{P} 3}+\Delta^{\mathrm{P} 4}+\Delta^{\mathrm{ISW}}$.

\section{FORECASTING FORMALISM}

The aim of this paper is to produce forecasts simultaneously for the detectability of the general relativistic corrections in terms of $\epsilon_{\mathrm{GR}}$ and for the level of primordial non-Gaussianity parametrized by $f_{\mathrm{NL}}$. We do so using the Fisher matrix approach, extending the formalism that was used in [15] to include different tracers.

\section{A. Fisher matrix with multiple tracers}

Let us consider an experiment targeting a number of tracers, each labeled by a Greek index $\alpha$; each tracer results in a number of maps of the sky, labeled by a Roman index $i$. For the purposes of this paper, these maps correspond to different redshift bins or frequency bands. All the cosmological information is contained in the harmonic coefficients for each tracer and map, which we label by a combined index $(\alpha, i)$ (i.e. $a_{\ell m}^{(\alpha, i)}$ are the harmonic coefficients for the $\alpha$ th tracer in its $i$ th redshift bin). For Gaussian random fields, the most important observable is the power spectrum, defined as the covariance of the $a_{\ell m}^{(\alpha, i)} \mathrm{s}$ :

$$
\left\langle a_{\ell m}^{(\alpha, i)}\left(a_{\ell^{\prime} m^{\prime}}^{(\beta, j)}\right)^{*}\right\rangle \equiv \delta_{\ell \ell^{\prime}} \delta_{m m^{\prime}} C_{\ell m}^{(\alpha, i)(\beta, j)} .
$$

We will assume that all tracers can be cleanly separated into signal and noise contributions $a_{\ell m}^{(\alpha, i)}=s_{\ell m}^{(\alpha, i)}+n_{\ell m}^{(\alpha, i)}$, and that both components are statistically independent. Furthermore, we will assume that the noise is uncorrelated between different tracers:

$$
\left\langle n_{\ell m}^{(\alpha, i)}\left(n_{\ell^{\prime} m^{\prime}}^{(\beta, j)}\right)^{*}\right\rangle \equiv N_{\ell m}^{(\alpha), i j} \delta_{\alpha \beta} \delta_{\ell \ell^{\prime}} \delta_{m m^{\prime}} .
$$

This assumption would not be correct, for instance, if the two tracers were two different but not disjoint galaxy populations. For most applications it is also safe to assume that the noise power spectrum is uncorrelated between different redshift bins. 
Arranging the combined indices $(\alpha, i)$ as a single one-dimensional index, we can write the $a_{\ell m}^{(\alpha, i)} \mathrm{s}$ and the power spectra as a vector $\mathbf{a}_{\ell m}$ and a matrix $\mathrm{C}_{\ell}$, respectively. For example, for two tracers $\alpha=\{1,2\}$ distributed over 2 and 1 redshift bins, respectively, we would have $\mathbf{a}_{\ell m}=\left(a_{\ell m}^{(1,1)}, a_{\ell m}^{(1,2)}, a_{\ell m}^{(2,1)}\right)$, and a power spectrum

$$
\mathrm{C}_{\ell}=\left(\begin{array}{cc|c}
C_{\ell}^{(1,1)(1,1)} & C_{\ell}^{(1,1)(1,2)} & C_{\ell}^{(1,1)(2,1)} \\
& C_{\ell}^{(1,2)(1,2)} & C_{\ell}^{(1,2)(2,1)} \\
\hline & & C_{\ell}^{(2,1)(2,1)}
\end{array}\right),
$$

where we have omitted the redundant components strictly below the diagonal. It is then easy to show that the Fisher matrix for the observable $\mathbf{a}_{\ell m}$ is the same as for the single tracer case, where the power spectrum matrix now contains also all possible cross-correlations between different tracers. Thus, for a set of parameters $\left\{\theta_{\mu}\right\}$, the covariance matrix $C_{\mu \nu} \equiv\left\langle\left(\theta_{\mu}-\bar{\theta}_{\mu}\right)\left(\theta_{\nu}-\bar{\theta}_{\nu}\right)\right\rangle$ can be approximated by the inverse of $F_{\mu \nu}$, where

$$
F_{\mu \nu}=f_{\text {sky }} \sum_{\ell=2}^{\ell_{\max }} \frac{2 \ell+1}{2} \operatorname{Tr}\left[\partial_{\mu} \mathrm{C}_{\ell} \mathrm{C}_{\ell}^{-1} \partial_{\nu} \mathrm{C}_{\ell} \mathrm{C}_{\ell}^{-1}\right]
$$

and $\partial_{\mu}$ is the partial derivative with respect to the $\mu$ th parameter. In our forecasts the derivatives were computed using central finite differences,

$$
\partial_{\mu} f=\frac{f\left(\theta_{\mu}+\delta \theta_{\mu}\right)-f\left(\theta_{\mu}-\delta \theta_{\mu}\right)}{2 \delta \theta_{\mu}}+\mathcal{O}\left(\delta \theta^{3}\right)
$$

where the intervals $\delta \theta_{\mu}$ are such that the derivatives converge to the required numerical accuracy.

As we pointed out above, we can divide the power spectrum up between two uncorrelated signal and noise contributions, $\mathrm{C}_{\ell}=\mathrm{C}_{\ell}^{S}+\mathrm{N}_{\ell}$. The cosmological power spectra $\mathrm{C}_{\ell}^{S}$ are given by Eq. (9), and were calculated using a modified version of the public CLASS code $[20,33]$. This version is based on the code used in [15], with added capability to handle an arbitrary number of tracers [34]. For our fiducial cosmology we adopted a model consistent with the best-fit flat $\Lambda \mathrm{CDM}$ parameters from Planck [35], given by $\left(\Omega_{M}, f_{b}, h, w, A_{s}, n_{s}\right)=(0.315,0.156,0.67,-1,2.46 \times$ $\left.10^{-9}, 0.96\right)$. We further set $f_{\mathrm{NL}}=0$, the value for Gaussian initial conditions, and $\epsilon_{\mathrm{GR}}=\epsilon_{\mathrm{WL}}=1$. In our forecasts we use these 9 free parameters, as well as a number of nuisance parameters associated with the bias functions of the tracers under study, which we describe below. Any priors on these parameters, correlated or not, were added as Gaussian priors to the bare Fisher matrix $\left[F_{\mu \nu} \rightarrow F_{\mu \nu}+\left(C^{\text {prior }}\right)_{\mu \nu}^{-1}\right]$. In particular for the cosmological parameters listed above (excluding $f_{\mathrm{NL}}, \epsilon_{\mathrm{GR}}$ and $\epsilon_{\mathrm{WL}}$ ) we used the covariance matrix estimated from the corresponding Planck MCMC chains. We must also note that, since $\epsilon_{\mathrm{GR}}$ and $\epsilon_{\mathrm{WL}}$ are only effective parameters, used to characterize the level of
TABLE I. Redshift bins used for the bias function nuisance parameters, for each tracer.

\begin{tabular}{lcc}
\hline \hline Tracer & \# bins & \multicolumn{1}{c}{ Bin edges } \\
\hline Intensity mapping (SKA1) & 4 & $0.0,0.7,1.3,2.0,3.0$ \\
Continuum survey (SKA1) & 4 & $0.0,0.5,1.0,2.5,5.0$ \\
Photometric (LSST), red & 4 & $0.0,0.35,0.7,1.05,1.4$ \\
Photometric (LSST), blue & 5 & $0.0,0.5,1.0,1.5,2.2,3.0$ \\
Photometric (DES), red & 4 & $0.0,0.3,0.6,0.9,1.2$ \\
Photometric (DES), blue & 4 & $0.0,0.4,0.8,1.2,1.5$ \\
\hline \hline
\end{tabular}

detection of the GR and lensing effects, when forecasting for $f_{\mathrm{NL}}$ we fix both $\epsilon_{\mathrm{GR}}$ and $\epsilon_{\mathrm{WL}}$ to their actual value of 1 instead of marginalizing over them.

The uncertainty with which a given parameter can be constrained usually depends on the largest and smallest accessible scales. The smallest angular scale included in

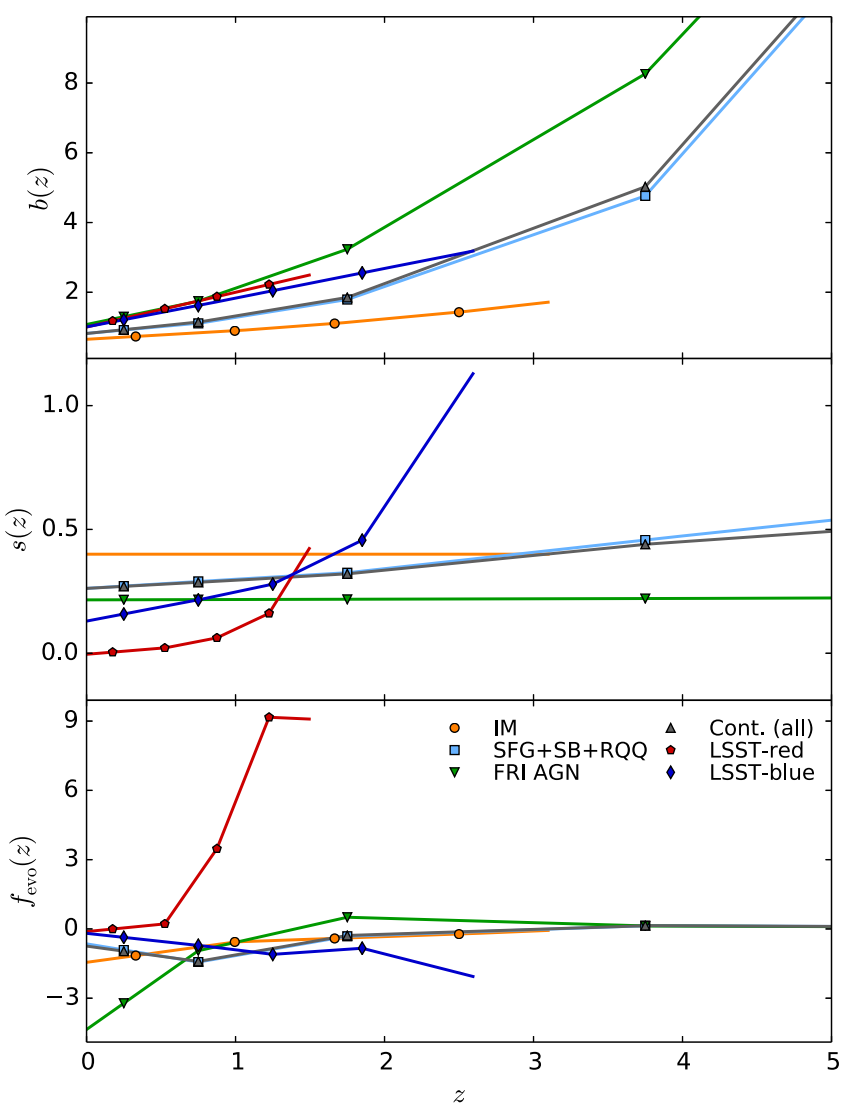

FIG. 1 (color online). Clustering bias (upper panel), magnification bias (middle panel) and evolution bias (lower panel) for the different tracers considered in this work: intensity mapping (orange), first continuum sample, made up of star-forming galaxies, starbursts and radio-quiet quasars (cyan), second continuum sample, made up of FRI-type active galactic nuclei (AGN) (green), combined continuum sample (grey), red LSST galaxies (red) and blue LSST galaxies (blue). In each curve, the markers show the nodes used as free parameters in the marginalization over these bias functions. Notice the very different evolution biases for red and blue galaxies. 
our forecasts is given by $\ell_{\max }$ in Eq. (18), while the smallest radial scale is given by the width of the redshift bins used in the analysis. These scales should be chosen on the basis of the angular and radial resolution of the experiment (e.g. the beam size for intensity mapping or the redshift uncertainty for photometric surveys), or in terms of the smallest scales for which we can trust our theoretical prediction (e.g. the nonlinear scale). Fortunately, since the observables we are interested in are mostly relevant on ultralarge scales, our forecasts are relatively insensitive to the choice of minimum scales. By default we use $\ell_{\max }=500$ for all the experiments considered here, and address the redshift binning scheme on an individual basis. We further assume that all these experiments will be able to probe angular scales down to $\ell_{\min }=2$, and we assess how the constraints degrade as a function of $\ell_{\min }$.

We treat the uncertainty in the bias functions $[b(z), s(z)$ and $f_{\text {evo }}(z)$ ] using the same method that was used in [15]: the mean value for each function is computed in a small set of wide redshift bins, and we use the linear interpolation between these values as the fiducial bias function passed to CLASS. We then treat each of the aforementioned mean values as free parameters that we include in our computation of the Fisher matrix and marginalize over. The redshift bins used to define these bias parameters for the different probes considered here are given in Table I, and the resulting bias functions are shown as a function of redshift in Fig. 1.

\section{B. Why multitracer surveys beat cosmic variance}

It was first noted in [16] that using different tracers deterministically related to the same density field it is

$$
\mathrm{C} \equiv\left(\begin{array}{c}
b_{1}^{2} \mathcal{P}\left(T^{\delta}+\beta_{1} \epsilon T^{G}\right)^{2}+N_{1} \\
b_{1}^{2} \mathcal{P}\left(T^{\delta}+\beta_{1} \epsilon T^{G}\right)\left(\gamma T^{\delta}+\beta_{2} \epsilon T^{G}\right)
\end{array}\right.
$$

where $\mathcal{P}$ is the power spectrum of $\Phi^{0}, \gamma \equiv b_{2} / b_{1}$, $\beta_{1} \equiv f_{1} / b_{1}, \beta_{2} \equiv f_{2} / b_{1}, N_{\alpha}$ is the noise power spectrum for each tracer and we have assumed that $\left\langle n_{1} n_{2}\right\rangle=0$. Note that we have omitted the dependence on the multipole order $\ell$.

We can estimate the best-case uncertainty on the amplitude $\epsilon$ by computing the inverse of its Fisher matrix element $\sigma^{2}(\epsilon) \simeq 1 / F_{\epsilon \epsilon}$. Using $F_{\epsilon \epsilon}=\frac{1}{2} \operatorname{Tr}\left[\left(\mathbf{C}^{-1} \partial_{\epsilon} \mathbf{C}\right)^{2}\right]$ we obtain

$$
\sigma^{2}(\epsilon) \simeq X_{2}\left[\frac{R+\beta_{1} \epsilon}{\beta_{2}-\gamma \beta_{1}}\right]^{2}+X_{1}\left[\frac{\gamma R+\beta_{2} \epsilon}{\beta_{2}-\gamma \beta_{1}}\right]^{2},
$$

where we have defined the ratios $X_{\alpha} \equiv N_{\alpha} /\left(\mathcal{P}\left(b_{1} T^{\delta}\right)^{2}\right)$ and $R \equiv T^{\delta} / T^{G}$, and we have linearized the result with respect to $X_{i}$. Several conclusions can be drawn from Eq. (23): possible to measure certain parameters with uncertainties smaller than the cosmic-variance limit. The aim of this section is to briefly describe the mechanism by which this is possible, and how we could benefit from it in order to detect the relativistic corrections presented in Sec. II A.

Let us consider a simplified scenario, where we measure the fluctuations in the source number counts of two different tracers $(\alpha=1,2)$ in the same single redshift bin. For the sake of argument let us assume that only two terms contribute to the total perturbation:

$$
a_{\ell m}^{\alpha}=b_{\alpha} \Delta_{\ell m}^{\delta}+f_{\alpha} \epsilon \Delta_{\ell m}^{G}+n_{\ell m}^{\alpha} .
$$

Here $\Delta^{\delta}$ and $\Delta^{G}$ are the terms corresponding the intrinsic perturbation in the matter density and a generic relativistic correction respectively, $b_{\alpha}$ is the clustering bias, $f_{\alpha}$ is a tracer-dependent bias function affecting the GR correction (i.e. similar to the evolution or magnification biases) and $n_{\ell m}^{\alpha}$ is a noise term. We have explicitly extracted the amplitude of the GR term as a parameter, $\epsilon$, equivalent to $\epsilon_{\mathrm{GR}}$. This can be rewritten as

$$
a_{\ell m}^{\alpha}=\left(b_{\alpha} T_{\ell}^{\delta}+f_{\alpha} \epsilon T_{\ell}^{G}\right) \Phi_{\ell m}^{0}+n_{\ell m}^{\alpha},
$$

where $T_{\ell}^{\delta}$ and $T_{\ell}^{G}$ are the transfer functions corresponding to the density and GR correction terms, and $\Phi_{\ell m}^{0}$ is the primordial perturbation.

The covariance matrix for both tracers in each multipole is

$$
\left.\begin{array}{c}
b_{1}^{2} \mathcal{P}\left(T^{\delta}+\beta_{1} \epsilon T^{G}\right)\left(\gamma T^{\delta}+\beta_{2} \epsilon T^{G}\right) \\
b_{1}^{2} \mathcal{P}\left(\gamma T^{\delta}+\beta_{2} \epsilon T^{G}\right)^{2}+N_{2}
\end{array}\right),
$$

(1) The error on $\epsilon$ is completely limited by the noise level, and not by cosmic variance. This could have been anticipated by looking at Eq. (21) in the noiseless case $\left(n^{\alpha} \rightarrow 0\right)$ : by taking the ratio $a_{\ell m}^{1} / a_{\ell m}^{2}$ it would be possible to cancel the stochasticity of $\Phi^{0}$ and determine $b_{\alpha}, f_{\alpha}$ and $\epsilon$ free of cosmic variance. This is the key to understanding the multitracer technique.

(2) If the amplitude of the relativistic correction is subdominant, (i.e. $R \gg 1$ ), as is the case for the terms studied here, the uncertainty in $\epsilon$ becomes proportional to $R$, and thus achieving $\sigma(\epsilon) \ll 1$ would still require very low noise levels and/or appropriate values for the bias parameters $f_{\alpha}$.

(3) The effect of the factor $\beta_{2}-\gamma \beta_{1}$ in the denominator is twofold. First, by choosing two tracers with very 
different bias functions (e.g. $f_{2} \gg f_{1}$ ) it is possible to further reduce the uncertainty on $\epsilon$. This is key to understand the results presented in subsequent sections. Second, in the case $\beta_{1}=\beta_{2}$ and $\gamma=1$ the uncertainty on $\epsilon$ diverges, which is a consequence of the fact that in this case both tracers are actually the same one, and nothing can be gained from treating them as being different.

Although this result is based on a toy model, it allows us to build a picture regarding the optimal tracers that should be used in order to achieve a measurement of the GR corrections: the ideal case would be to combine measurements of two (or more) high signal-to-noise tracers with very different magnification and/or evolution biases.

\section{OPTICAL AND NEAR-INFRARED EXPERIMENTS}

\section{A. Photometric redshift surveys}

The observational study of cosmological large-scale structure has so far been dominated by galaxy surveys performed in optical and infrared wavelengths [36-38]. In the ideal case, redshifts and angular positions are measured for a homogeneous sample of targets, and the threedimensional matter density distribution is probed through the fluctuations in the galaxy number density. Accurate redshift measurements are, however, very time consuming, especially for faint sources, and therefore spectroscopic redshifts are usually only available for a subsample of all the photometrically detected sources. This has two critical drawbacks: first, it prevents spectroscopic redshift surveys from covering the largest accessible scales, which is key to studying the observables described in Sec. II. Second, the subsampling of the galaxy population produces relatively high shot-noise levels, which dominate the error budget for parameters like $f_{\mathrm{NL}}$ and $\epsilon_{\mathrm{GR}}$ in the multitracer scheme. These problems can be partially circumvented using photometric redshifts (photo- $z$ s), i.e. redshifts inferred from the observed source fluxes in a number of broadbands. On the one hand, these redshift estimates are much less accurate than their spectroscopic counterparts, which limits the ability of photometric redshift surveys to study clustering statistics on small radial scales. On the other hand, photo$z \mathrm{~s}$ can, in principle, be estimated for all the sources detected above a given flux threshold, and thus the problems of volume coverage and shot noise are greatly alleviated. Since the observables studied here are only relevant on the largest scales, and their measurement can be optimized by reducing the noise levels of the experiment, photometric redshift surveys seem to be ideally suited for our purposes.

We will focus our discussion on the Large Synoptic Survey Telescope (LSST, [39]), since it represents the widest $\left(\sim 20000 \mathrm{deg}^{2}\right)$ and deepest $(r \sim 27)$ photometric survey planned in the foreseeable future, but we will also present forecasts for the ongoing Dark Energy Survey [40].

In this section we focus on the advantages of combining clustering information for different galaxy samples observed within the same experiment. In particular we will use a simplified picture in which we assume that the full galaxy population can be separated into two disjoint samples of "red" and "blue" galaxies:

(i) Red galaxies are usually identified with an early population of galaxies where star formation has ended. This produces a red spectrum, usually characterized by a deep Balmer/4000 $\AA$ break. This spectral type is also commonly associated with an elliptical morphology, supported by random motions rather than angular momentum, possibly caused by mergers. The red population appears only after the peak in the star formation rate $(z \sim 1.9)$, and its comoving number density is known to decrease sharply at redshifts larger than $\sim 1$.

The simplest models of galaxy formation suggest that the earliest galaxies should have formed in the peaks of the density field. Thus the red population can be associated with larger-mass halos and a larger clustering bias.

(ii) Blue galaxies, on the other hand, are characterized by a population of young stars, showing strong Balmer emission lines. They are usually associated with a spiral morphology, smaller halo mass and lower clustering bias. The number density of blue galaxies shows no sharp increase or decrease, and LSST should be able to observe them up to much higher redshifts than the red population.

This simplified model of the galaxy population is based on the observed bimodal nature of the color-magnitude diagram [41]. The actual picture is nowhere as simple (e.g. red spirals and blue ellipticals are known to exist), but this model allows us to study the potential improvement in the figure of merit for ultralarge-scale observables caused by combining clustering observations for two populations with similar characteristics.

Of particular interest would be the sharp decay in the number density of red galaxies. As was shown in [15], this is associated with a large evolution bias, and enhances the amplitude of the relativistic corrections described in Sec. II A, although this enhancement was shown to be too small to grant a detection of these effects using single-tracer observations. However, as discussed in Sec. III B, combining red and blue galaxies, the latter having a much lower evolution bias, could potentially boost the figure of merit for $\epsilon_{\mathrm{GR}}$.

As was done in [15], we base our estimates for the number densities of both populations, as well as their magnification and evolution biases on luminosity functions estimated by $[42,43]$ in the $r$-band. The details of this model are summarized in [15]. Regarding the clustering 
bias, we parametrize its redshift dependence for the red and full galaxy samples as:

$$
b_{\text {red }}(z)=1+z, b_{\text {full }}(z)=1+0.84 z,
$$

based on the simulations of [44] and the measurements of [45]. We then assume that the clustering bias of the full sample is a weighted average of the red and blue samples, and compute the clustering bias of the latter as:

$$
b_{\text {blue }}(z)=\frac{\bar{n}_{\text {full }}(z) b_{\text {full }}(z)-\bar{n}_{\text {red }}(z) b_{\text {red }}(z)}{\bar{n}_{\text {blue }}(z)} .
$$

Note that this result stems simply from the assumption that the full galaxy population is composed of red and blue galaxies only.

Finally we assume a magnitude cut of $i=25.3(r \simeq 26)$, corresponding to the so-called "gold sample," and a sky fraction $f_{\text {sky }}=0.5\left(\sim 20000 \mathrm{deg}^{2}\right)$ for LSST. We find that the total number counts as well as the magnitude distribution for each galaxy type obtained using these models is in good agreement with previous estimates $[39,46]$. For the Dark Energy Survey we will assume a magnitude limit $r=24$ and a sky fraction of $0.125\left(\sim 5000 \mathrm{deg}^{2}\right)$.

The redshift binning scheme was determined for both samples in terms of their expected photometric redshift errors. We assume Gaussianly distributed errors with a variance $\sigma_{z}(z)=\sigma_{0}(1+z)$. The photo- $z$ requirements for LSST as quoted in [39] are $\sigma_{0}<0.05$ with a goal of 0.02 . Since the spectral characteristics of red galaxies, particularly the prominent Balmer break, make their photo- $z \mathrm{~s}$ more reliable, we assumed that the goal quoted above will be attained for this sample, and we conservatively set the photo- $z$ error parameter for blue galaxies to the requirement limit. I.e.:

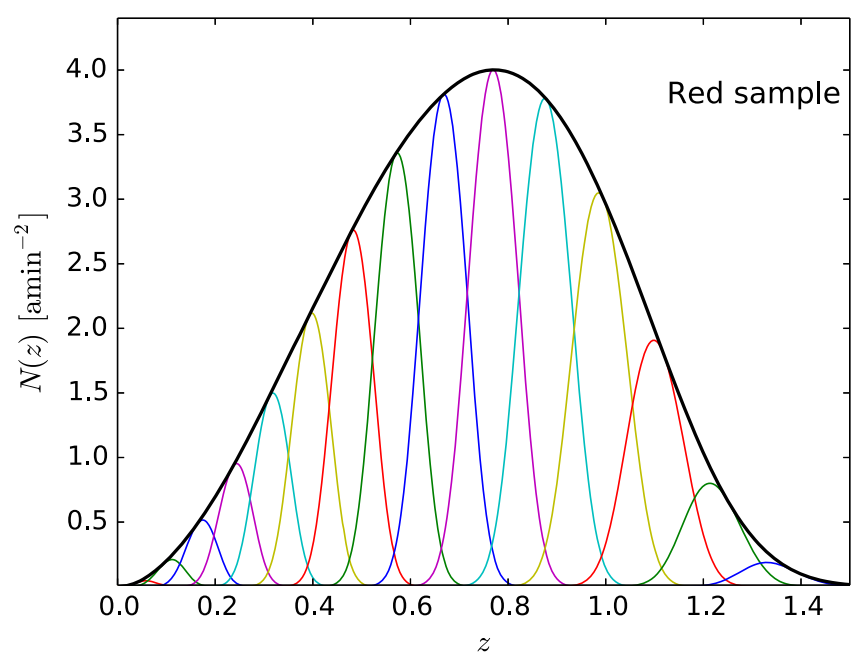

$$
\sigma_{0}^{\text {red }}=0.02, \quad \sigma_{0}^{\text {blue }}=0.05 .
$$

For DES we kept $\sigma_{0}^{\text {blue }}=0.05$ but increased the error parameter for red galaxies to $\sigma_{0}^{\text {red }}=0.03$, since the quality of the photo- $z \mathrm{~s}$ should be degraded by the absence of the $u$-band in the DES filter set. We define the redshift bins used for each sample as top-hat bins in the space of photo$z$ s with a width three times the size of the photo- $z$ uncertainty at the median redshift of the bin. This width was chosen in order to reduce the correlations between redshift bins caused by the tails of the photo- $z$ probability distribution. Thus, a top-hat bin in photo- $z$ space with edges $z_{0}$ and $z_{f}$ has a true- $z$ window function given by

$$
W(z) \propto \bar{N}(z) w\left(z \mid z_{0}, z_{f}\right),
$$

where $w\left(z \mid z_{0}, z_{f}\right)$ is the integral of the photo- $z$ distribution over the redshift bin. For the Gaussian photo- $z$ errors assumed here, $w$ takes the form

$$
w\left(z \mid z_{0}, z_{f}\right)=\frac{1}{2}\left[\operatorname{erf}\left(\frac{z-z_{0}}{\sqrt{2} \sigma_{z}}\right)-\operatorname{erf}\left(\frac{z-z_{f}}{\sqrt{2} \sigma_{z}}\right)\right] .
$$

Using this prescription we divide the red and blue samples into 15 and 9 redshift bins for LSST (10 and 7 bins respectively for DES), shown in Fig. 2 together with their overall redshift distribution.

The noise power-spectrum is given by $N_{\ell}^{i j}=\delta_{i j} / n^{i}$, where

$$
n^{i} \equiv \int_{0}^{\infty} d z \bar{N}(z) w^{i}(z)
$$

is the angular number density of sources in the $i$ th redshift bin with a photo- $z$ window function $w^{i}$, given by Eq. (28).

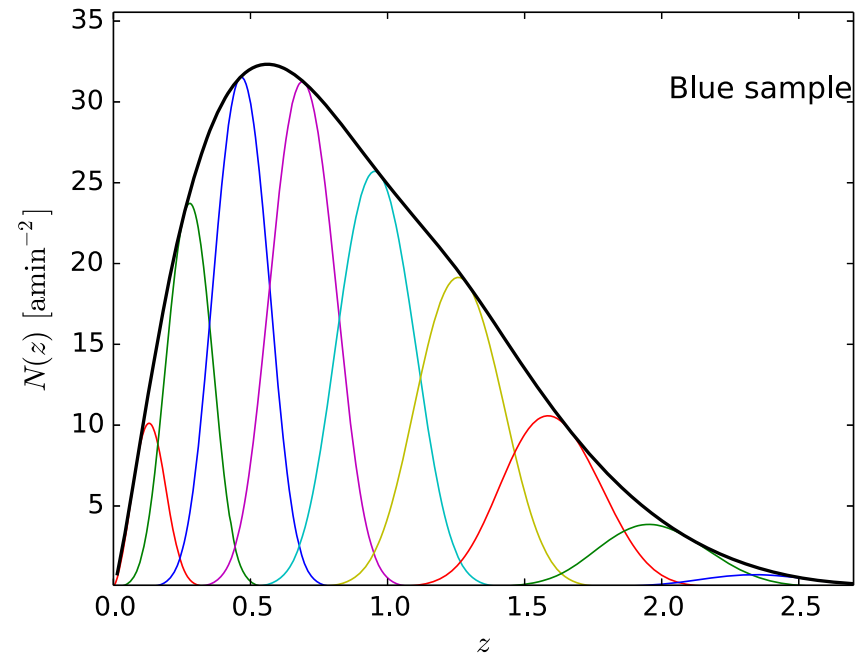

FIG. 2 (color online). Redshift distribution (thick solid black lines) and radial window functions (thin colored lines) for the redshift bins used here for the red (left panel) and blue (right panel) galaxy populations for LSST. 


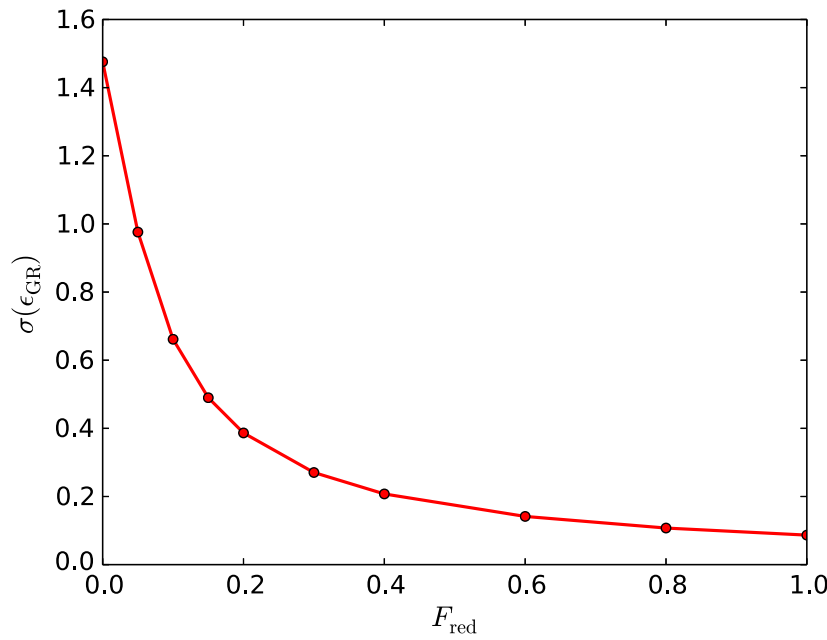

FIG. 3 (color online). Improvement of the detection level for the relativistic corrections as a function of $F_{\text {red }}$ [see Eq. (30)], which parametrizes the amplitude of the difference in the values of $f_{\text {evo }}$ and $s$ between red and blue galaxies. As discussed in Sec. III B the uncertainty on $\epsilon_{\mathrm{GR}}$ decreases as the values of these two parameters for both populations become more different.

\section{B. Forecasts}

We begin our discussion with an assessment of the detectability of the GR effects in the simplest and most optimistic case: we marginalize only over $\epsilon_{\mathrm{GR}}$ and $f_{\mathrm{NL}}$ (since the latter could potentially be very degenerate with the former) and fix all other parameters to their fiducial values. This would represent what an experiment aiming at a first detection of the GR corrections would do. The results using the red sample only, the blue sample only and the combination of both (in a multitracer sense) are

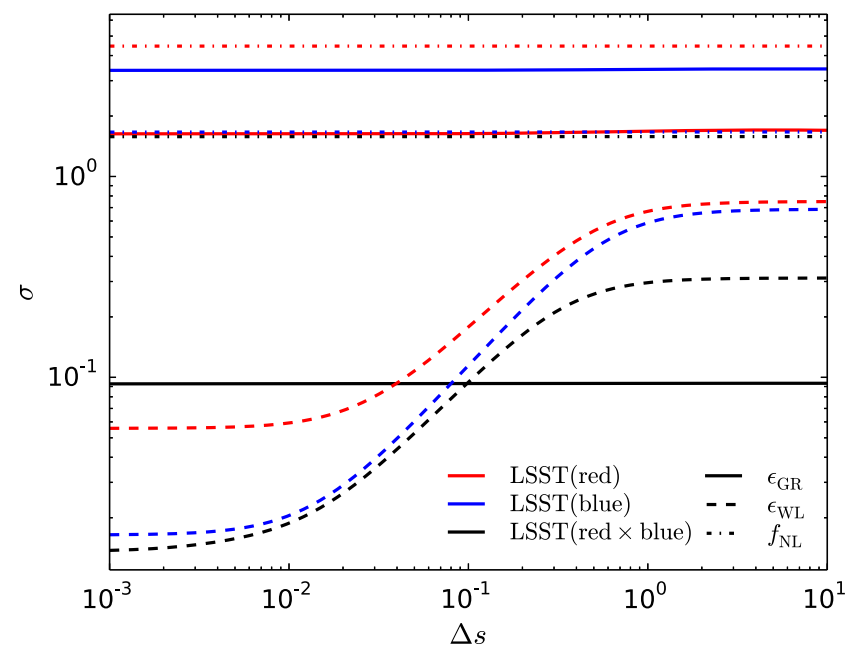

$$
\begin{aligned}
\sigma_{\text {red }}\left(\epsilon_{\mathrm{GR}}\right) & =1.531, \quad \sigma_{\text {blue }}\left(\epsilon_{\mathrm{GR}}\right)=3.094, \\
\sigma_{\text {joint }}\left(\epsilon_{\mathrm{GR}}\right) & =0.095 .
\end{aligned}
$$

We can see that the combination of both samples causes a dramatic improvement in the figure of merit. From our discussion in Sec. III B, we can a priori attribute this to two causes: the low shot-noise levels for LSST and the very different bias parameters of blue and red galaxies, especially the evolution bias, as can be seen in Fig. 1.

In order to show this explicitly we have carried out the following exercise. We recomputed our forecasts for a fictitious LSST experiment with an ordinary blue sample and a red sample with the same number density and clustering bias shown in Figs. 2 and 1, but with magnification and evolution biases given in terms of a parameter $F_{\text {red }}$ as:

$$
\begin{aligned}
s^{\text {red }} & \rightarrow F_{\text {red }} s^{\text {red }}+\left(1-F_{\text {red }}\right) s^{\text {blue }}, \\
f_{\text {evo }}^{\text {red }} & \rightarrow F_{\text {red }} f_{\text {evo }}^{\text {red }}+\left(1-F_{\text {red }}\right) f_{\text {evo }}^{\text {blue }} .
\end{aligned}
$$

For a value of $F_{\text {red }}=0$ both samples would have the same magnification and evolution biases and, according to our discussion in Sec. III B the uncertainty on $\epsilon_{\mathrm{GR}}$ would not suffer any significant enhancement. As we increase the value of $F_{\text {red }}$ toward 1 we should gradually recover the result quoted above. This is explicitly shown in Fig. 3, which presents the forecasted uncertainty on $\epsilon_{\mathrm{GR}}$ as a function of $F_{\text {red }}$. A $2 \sigma$ detection of $\epsilon_{\mathrm{GR}}$ would already be achievable, in this optimistic setup, just with $F_{\text {red }} \sim 0.2$.

Since it seems like it could be possible to detect the relativistic corrections using the multitracer method, we must now explore the possible degeneracies that could, on the one hand, preclude this detection and, on the other,

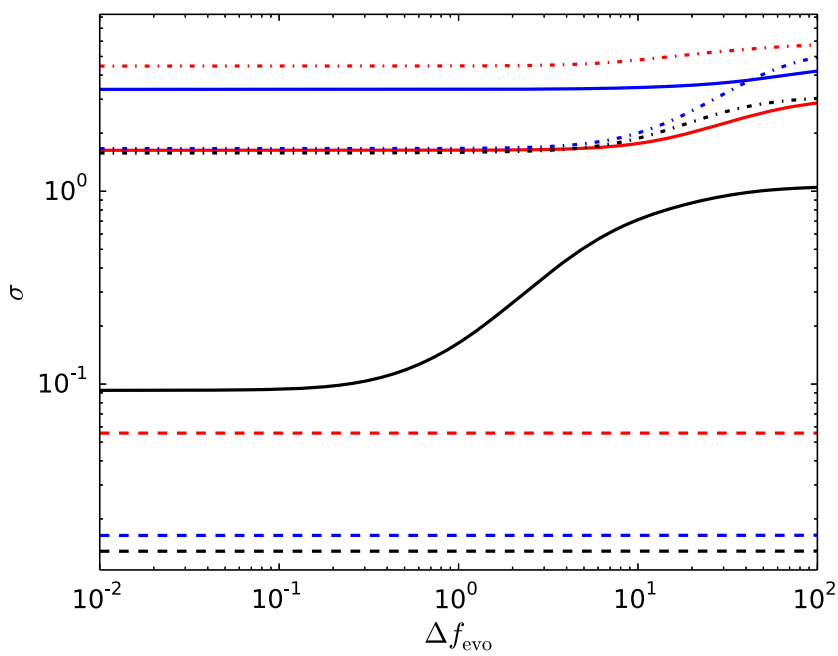

FIG. 4 (color online). Dependence of the uncertainty on $f_{\mathrm{NL}}, \epsilon_{\mathrm{GR}}$ and $\epsilon_{\mathrm{WL}}$ on the assumed Gaussian constant prior on the magnification bias (left panel) and the evolution bias (right panel). The significance of the detection of the lensing magnification term depends very strongly on the prior on $s$. Likewise the error on $\epsilon_{\mathrm{GR}}$ is particularly sensitive to the prior on $f_{\text {evo }}$ when both tracers are combined, and the detection of GR effects only becomes optimal for $\Delta f_{\text {evo }} \lesssim 0.1$. 


\section{ALONSO AND P. G. FERREIRA}

affect the measurements of other parameters. We do not observe strong degeneracies between $\epsilon_{\mathrm{GR}}$ and any cosmological parameter, and only a very mild one with $f_{\mathrm{NL}}$. The situation is different, however, for the nuisance bias parameters. Since the amplitude of the relativistic corrections is partially determined by the values of the magnification and evolution biases of the samples under study, it is probably very difficult to claim a detection of these effects without good prior information on $s$ and $f_{\text {evo }}$. We have studied the dependence of the uncertainties on $f_{\mathrm{NL}}$, $\epsilon_{\mathrm{GR}}$ and $\epsilon_{\mathrm{WL}}$ on a Gaussian prior, constant in redshift, for these two bias parameters. The results are shown in Fig. 4 in the same three cases explored before (red and blue samples independently and jointly). As should be obvious, the detection level of the lensing magnification term depends critically on the precision with which the magnification bias is known, and an error on $s(z)$ of the order of $\sim 0.01$ would be necessary to optimize this measurement. On the other hand both $f_{\mathrm{NL}}$ and $\epsilon_{\mathrm{GR}}$ are almost insensitive to the prior on $s$. The situation is different for $f_{\text {evo }}$ : both $f_{\mathrm{NL}}$ and $\epsilon_{\mathrm{GR}}$ are sensitive to the prior on $f_{\text {evo }}$, although only a very broad prior of $\Delta f_{\text {evo }}=\mathcal{O}(1)$ would be necessary to optimize their measurement in the single-tracer case. For the multitracer analysis, however, the detection of the GR corrections depends critically on having precise enough measurements of the evolution bias, which as we saw is responsible for significantly boosting the signal. The optimal case would correspond to a precision of $\Delta f_{\text {evo }} \lesssim 0.1$. We have also studied the possible dependence of the uncertainties on our key observables with the priors on the clustering bias, finding no significance degeneracies.

The availability of such tight priors on the evolution and magnification biases will depend directly on our ability to model the joint redshift and magnitude distribution of the galaxy samples under study. This could be challenging for photometric redshift surveys, given the poor redshift uncertainties, and would probably entail relying on external data sets or on a spectroscopic follow-up of a representative subsample of the survey. In order to gain some intuition regarding the feasibility of these measurements we have estimated the uncertainty on the evolution bias of red galaxies (responsible for the enhanced amplitude of the GR effects) given the uncertainties in the measurements of the luminosity function of red galaxies of [42] on which our model is based. In the high-redshift end of the distribution we obtain an uncertainty $\Delta f_{\text {evo }} \sim 2$, which decreases toward smaller redshifts as the number counts increase. In the future these uncertainties should improve significantly due to the availability of larger and deeper data sets, and therefore we will present our forecasts in a pessimistic and an optimistic scenario, where we will assume the priors:

$$
\begin{aligned}
\text { Pessimistic } & \rightarrow \Delta s=\Delta f_{\text {evo }}=1, \\
\text { Optimistic } & \rightarrow \Delta s=\Delta f_{\text {evo }}=0.1 .
\end{aligned}
$$

PHYSICAL REVIEW D 92, 063525 (2015)

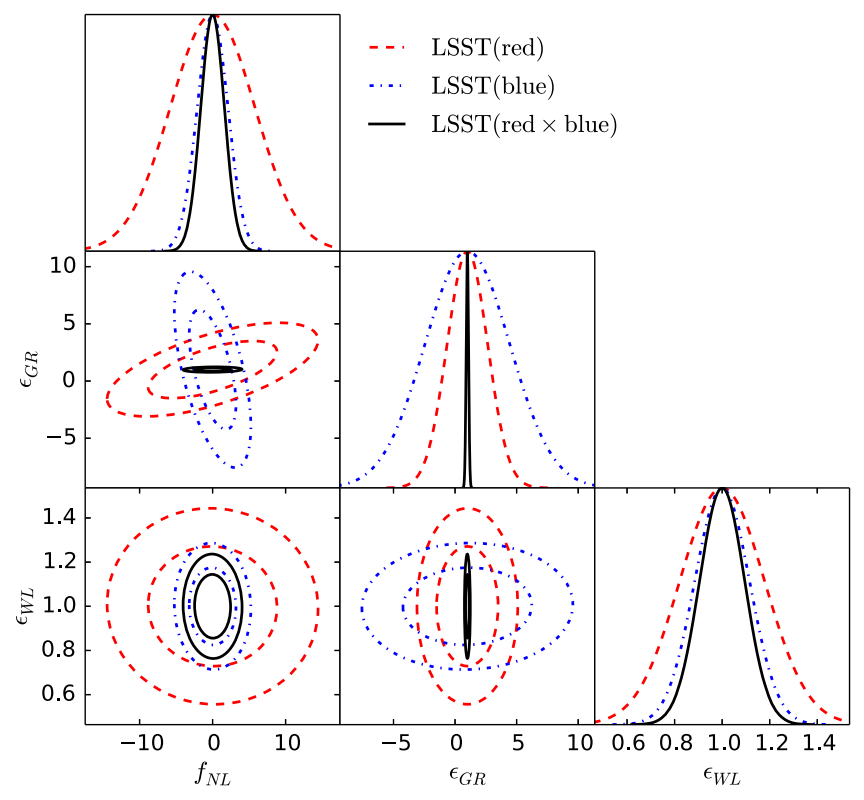

FIG. 5 (color online). Joint constraints on $f_{\mathrm{NL}}, \epsilon_{\mathrm{GR}}$ and $\epsilon_{\mathrm{WL}}$ for the red and blue samples (red and blue contours, respectively) as well as for a joint analysis of both (black contours) for optimistic priors $\Delta f_{\text {evo }}=\Delta s=0.1$.

Figure 5 presents the joint forecasted uncertainties on $f_{\mathrm{NL}}, \epsilon_{\mathrm{GR}}$ and $\epsilon_{\mathrm{WL}}$ for LSST in the optimistic scenario for the three cases mentioned above: red-only (red), blue-only (blue) and joint analysis (black). The numerical values for these uncertainties are given in Table II. Note that, unlike in the case of $\epsilon_{\mathrm{GR}}$ there is only a mild improvement in the figure of merit for $f_{\mathrm{NL}}$ and $\epsilon_{\mathrm{WL}}$ in the multitracer analysis with respect to the deepest LSST blue sample alone. The main reason for this is that, unlike in the case of the evolution bias, the differences in the magnification and clustering biases of both tracers are not so large.

We have also produced forecasts for the Dark Energy Survey, using the same models adopted for LSST with a magnitude limit of $r=24$ and $f_{\text {sky }}=1 / 8$. The results are also included in Table II: DES should, in the best-case scenario, be able to make a $\sim 3 \sigma$ detection of the relativistic corrections. This result, however, could be compromised by the possible systematic effects that could dominate the clustering statistics on large angular scales. We will discuss these in Sec. VII.

\section{RADIO EXPERIMENTS}

\section{A. Cosmological radio surveys}

With the forthcoming wide-area radioastronomy facilities, the field of observational large-scale structure will soon begin to reap the benefits of observing in the radio regime. The low atmospheric absorption and dust obscuration in a wide range of radio frequencies makes it possible to observe objects at significantly higher redshifts than are usually targeted in optical/near infrared surveys, and in the 
TABLE II. Forecasted constraints on $f_{\mathrm{NL}}, \epsilon_{\mathrm{GR}}$, and $\epsilon_{\mathrm{WL}}$ for the different experiments explored in this work. In each case, the constraints assuming optimistic and pessimistic priors on the magnification and evolution biases are shown with and without parentheses, respectively. $\left(^{*}\right)$ Note that the uncertainty on $\epsilon_{\mathrm{WL}}$ is shown to be smaller for DES than LSST. This does not mean that DES would be better at measuring lensing, only that the value of the magnification bias at the magnitude limit of DES ( 24) enhances the lensing magnification effect. LSST would be able to greatly improve on this result by adopting a different (lower) flux cut. This was already noted in [47].

\begin{tabular}{lllll}
\hline \hline Experiment type & \multicolumn{1}{c}{ Tracers } & $\sigma\left(f_{\mathrm{NL}}\right)$ & $\sigma\left(\epsilon_{\mathrm{GR}}\right)$ & $\sigma\left(\epsilon_{\mathrm{WL}}\right)$ \\
\hline Photometric survey & LSST, red-only & $4.53(4.54)$ & $1.65(1.70)$ & $0.18(0.67)$ \\
(LSST) & LSST, blue-only & $1.71(1.72)$ & $3.45(3.48)$ & $0.12(0.61)$ \\
& LSST, red $\times$ blue & $1.62(1.63)$ & $0.10(0.17)$ & $0.10(0.36)$ \\
& DES, red $\times$ blue & $7.18(7.20)$ & $0.29(0.32)$ & $0.06(0.06)\left({ }^{*}\right)$ \\
Radio & IM-only & $3.00(3.01)$ & $2.71(2.75)$ & $1.47(1.95)$ \\
(SKA1-MID) & IM $\times$ Cont., 1 sample & $0.86(0.89)$ & $1.23(1.43)$ & $0.33(2.21)$ \\
& IM $\times$ Cont., 2 samples & $0.69(0.71)$ & $23.9(31.1)$ & $0.56(2.85)$ \\
Synergy & Continuum-only, 2 samples & $1.91(1.97)$ & $0.15(0.39)$ & $0.09(0.32)$ \\
(SKA1-MID $\times$ LSST) & IM $\times$ all & $0.41(0.41)$ & $0.05(0.12)$ & $0.07(0.19)$ \\
\hline \hline
\end{tabular}

next decades radio surveys will be able to cover comparably wide areas with similar source number densities. In addition to that, the relative isolation of the few emission lines of astrophysical interest in the radio spectrum (e.g. the neutral hydrogen line at $1.4 \mathrm{GHz}$ or molecular $\mathrm{CO}$ at $115 \mathrm{GHz}$ ) makes it possible to conduct intensity mapping observations, producing tomographic maps of the density fluctuations of these species.

In this section we will describe two main cosmological probes of the low-redshift Universe in radio experiments: intensity mapping (IM) of neutral hydrogen (HI) and continuum surveys. Both probes have complementary properties that make their cross-correlation extremely interesting: while intensity mapping is able to map the density field with remarkable radial (frequency) resolution, but only on fairly big angular scales, continuum surveys lack any radial information, but are able to resolve individual sources. On the other hand, while HI is known to be a very faithful tracer of the matter density (i.e. has a low clustering bias), many radio sources, such as AGNs, are highly biased, and thus, as discussed in Sec. III B, their combination could be a useful way to measure the level of primordial nonGaussianity below the cosmic variance limit.

For both probes we produce our forecasts assuming the current design for Phase 1 of the Square Kilometre Array (SKA) $[48,49]$. In both cases we assume that the experiment of choice will be SKA1-MID, an array of 200 single-pixel, $15 \mathrm{~m}$ dishes to be installed in South Africa. It will cover the frequency range $350-1760 \mathrm{MHz}(z \lesssim 3)$ in two separate bands. Note that there are other intensity mapping experiments planned for the future, such as CHIME [50] or BINGO [51]. However, the SKA should provide a better coverage of the largest angular scales, which is the topic of this work. Additionally, the SKA is a multiscience facility that will perform both intensity mapping and continuum observations, and therefore cross - correlating both data sets from the same experiment should be more straightforward.

\section{Intensity mapping}

While it is, in principle, possible to determine the radial position of radio sources through the redshift of the $21 \mathrm{~cm}$ line, the intensity of this line from individual sources is very small, and long integration times are needed in order to resolve it with sufficiently high signal-to-noise ratio. For a fixed amount of observation time, this limits the source number density, depth and area that can be achieved with such surveys. Intensity mapping [52-54] is a relatively novel technique that aims to circumvent this problem by measuring the combined emission from all the sources in relatively wide pixels simultaneously at different frequencies. Adding up the emission from hundreds or thousands of sources it is possible to detect the large-scale fluctuations in the HI density field in three dimensions up to relatively large depths. This is achieved at the cost of losing potentially valuable information on small angular scales, since individual sources are not resolved, although this is not necessarily an issue if we target large-scale observables, as is the case of this work. As was shown in [15], however, two facts conspire against $\mathrm{HI}$ as a single-probe of non-Gaussianity and GR corrections: its relatively low clustering bias and the cancellation of the linear perturbations on angular distances, respectively.

We model the clustering, magnification and evolution biases for $\mathrm{HI}$ intensity mapping using the same approach described in Sec. IV B of [15], based on using a scaling relation between $\mathrm{HI}$ and halo mass of the form $M_{\mathrm{HI}}(z, M) \propto M^{\alpha}$, with $\alpha \simeq 0.6$. This allows us to estimate the HI density [and hence its evolution bias using Eq. (11)], as well as its clustering bias. As noted in Sec. II A, the exact cancellation of the perturbations on transverse distances for intensity mapping implies an effective magnification bias $s_{\mathrm{HI}}=2 / 5$. 
As described in [55], the most efficient use of SKA1MID for intensity mapping will be in single-dish mode, and we base our noise model on this configuration. For an autocorrelation experiment, and assuming no correlations between the noise in different frequency channels, the noise power spectrum is constant on all scales:

$$
N_{\ell}^{\mathrm{HI}, i j}=\delta_{i j} \sigma_{i}^{2},
$$

where $\sigma_{i}^{2}$ is the noise variance per steradian in the $i$ th frequency channel, which can be related to the survey characteristics as

$$
\sigma_{i}^{2}=\frac{T_{\text {sys }}^{2}\left(\nu_{i}\right) 4 \pi f_{\text {sys }}}{\delta \nu t_{\text {tot }} N_{\text {dish }}},
$$

where $t_{\text {tot }}$ is the total observation time, $f_{\text {sky }}$ is the observed sky fraction, $N_{\text {dish }}$ is the number of dishes in the experiment, $\delta \nu$ is the frequency bandwidth of the bin and $T_{\text {sys }}=T_{\text {sky }}+T_{\text {inst }}$ is the system temperature, which receives contributions both from the instrumental noise temperature and from the atmospheric and background noise $\left(T_{\text {sky }} \sim 60 \mathrm{~K} \times(\nu / 300 \mathrm{MHz})^{-2.5}\right)$.

Regarding the radial binning, we follow the procedure used in [15], subdividing the redshift range $(0 \leq z \leq 3)$ into intervals of equal comoving width. We produced forecasts for gradually thinner bins until the uncertainties on our large-scale parameters, $f_{\mathrm{NL}}$ and $\epsilon_{\mathrm{GR}}$ converged. We thus obtain our final forecasts for 100 frequency bins with a comoving width $\delta \chi \simeq 44 \mathrm{Mpc} / h$.

Since, in intensity mapping, sources are not detected individually, we must incorporate the effect of the telescope beam, and thus our model for the intensity mapping power spectrum is

$$
C_{\ell}^{i j}=C_{\ell}^{S, i j} B_{\ell}^{i} B_{\ell}^{j}+N_{\ell}^{i j},
$$

where $B_{\ell}^{i}$ is the harmonic transform of the instrumental beam in the $i$ th frequency bin. For this work we have assumed that the beams are isotropic and Gaussian: $B_{\ell}^{i}=\exp \left(-\ell(\ell+1) \theta_{B}^{2} / 2\right)$, with $\theta_{B}(\nu)=c /\left(2.35 \nu D_{\mathrm{dish}}\right)$, where $D_{\text {dish }}$ is the dish diameter. Note that when crosscorrelating intensity mapping with any discrete tracer $\alpha$, the effect of the beam is

$$
C_{\ell}^{(\mathrm{HI}, i)(\alpha, j)}=B_{\ell}^{i} C_{\ell}^{S,(\mathrm{HI}, i)(\alpha, j)} .
$$

The experiment parameters used for SKA1-MID were $T_{\text {inst }}=25 \mathrm{~K}, \quad f_{\text {sky }}=0.75\left(\sim 30000 \mathrm{deg}^{2}\right), \quad t_{\text {tot }}=10^{4} \mathrm{~h}$, $N_{\text {dish }}=200$ and $D_{\text {dish }}=15 \mathrm{~m}$.

\section{Continuum surveys}

The radio spectrum of most astrophysical sources is generally smooth and featureless, dominated by radio synchrotron emission with, perhaps, very few emission lines such as the aforementioned $21 \mathrm{~cm}$ signal. It is thus possible to integrate the flux from individual sources on rather wide frequency bands without losing too much information, while gaining access to much fainter sources than it would be possible to detect otherwise. Continuum surveys can therefore observe radio galaxies over incredibly large volumes with the caveat that all information regarding the radial distribution and clustering of these sources is completely unavailable.

A number of papers have already shown the power of continuum surveys to constrain the level of primordial nonGaussianity, especially making use of the multitracer technique [12]. Our aim here is to add on this result by considering the different cross-correlations with intensity mapping, as well as the potential of these cross-correlations to detect relativistic effects.

Our models for the redshift distribution and bias functions for the different radio sources considered here follow the method discussed in Appendix B of [15], based on the measurements of the luminosity functions by [56-58], used in [59] to produce semiempirical simulations for radio surveys. We will consider here four different galaxy populations: normal star-forming galaxies, starbursts, radio-quiet AGN and radio-loud AGN of the FR-I type. Owing to their very small number density, we omitted FRII galaxies from this analysis. Note that, as described in [12], separating the combined sample of radio galaxies into these four different types could be a challenging task, especially given the enormous size of the sample that the SKA will be able to collect. Furthermore, although it should be possible to distinguish radio-loud AGN from other sources on a morphological basis, differentiating radio-quiet quasars would probably require $\mathrm{X}$-ray observations with a different experiment. Further separating normal star-forming galaxies from starbursts would require optical and redshift information, which would be difficult to obtain for the whole redshift range. For this reason we produce our forecasts for two cases: a pessimistic scenario with no source differentiation (i.e. one single tracer) and an optimistic one with two disjoint samples, given by radio-loud AGN on the one hand and a combination of star-forming galaxies, starbursts and radioquiet quasars on the other. We will call these two samples "sample 2" and "sample 1," respectively.

As for intensity mapping, we consider here a SKA1-MID configuration, which should be able to detect radio sources to $z \sim 5$ over three quarters of the sky by integrating their continuum flux in the band $350-1050 \mathrm{MHz}$ with a rms noise of $S_{\mathrm{rms}}=1 \mu \mathrm{Jy}$, and we will assume a $5 \sigma$ detection threshold (i.e. $S_{\text {cut }}=5 \mu \mathrm{Jy}$ ). The redshift distribution of the two populations considered here as well as the combined sample for this flux cut can be seen in Fig. 6, and the corresponding clustering, magnification and evolution biases are shown in Fig. 1, together with those for HI intensity mapping. 


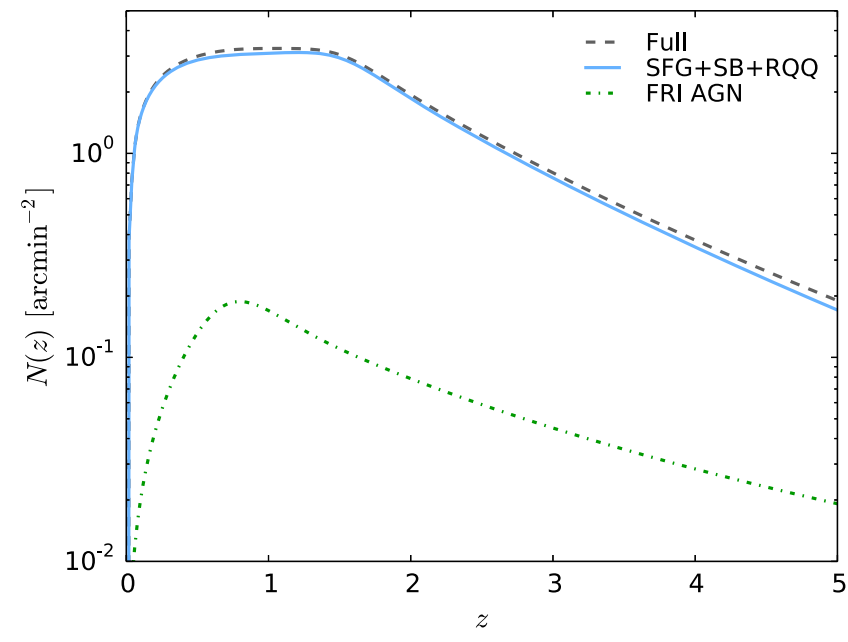

FIG. 6 (color online). Redshift distribution of radio sources for a flux cut $S<5 \mu \mathrm{Jy}$. The distribution for the combined population is shown in dark grey, while the two subsamples considered here are plotted in blue (sample 1-star-forming galaxies, starbursts and radio-quiet AGN) and green (sample 2-FRI AGN).

The noise power spectrum for continuum surveys is simply given by Poisson shot-noise $N_{\ell}^{\text {cont }}=\bar{N}_{\Omega}^{-1}$, where $\bar{N}_{\Omega}$ is the angular number density of sources (per steradian) in the survey. We must note that the intensity mapping noise is not completely uncorrelated with that of continuum sources. At low redshifts most of the HI is located inside galaxies, and so many of the galaxies contributing to the $\mathrm{HI}$ line emission will also be detected in the continuum survey. However, the contribution of this Poisson term to the total noise power spectrum is very small compared to the instrumental noise levels [55], and therefore we neglect these correlations.

\section{B. Forecasts}

We can expect the advantages of combining intensity mapping and continuum observations in a multitracer sense to be threefold: first, the large clustering bias of e.g. AGN combined with the very low $b$ of neutral hydrogen should significantly boost the amplitude of the $f_{\mathrm{NL}}$ signal. Second, the absence of perturbations on transverse scales for intensity mapping should help to separate the GR effects and lensing magnification contributions in the cross-correlation. Finally, the main drawback of continuum surveys is the absolute lack of radial information, and therefore, given the good redshift resolution of intensity mapping, combining both data sets should significantly improve any constraints.

We have computed the forecasted errors on $f_{\mathrm{NL}}, \epsilon_{\mathrm{GR}}$ and $\epsilon_{\mathrm{WL}}$ for four different tracer combinations:

(i) Case A: using only intensity mapping (IM only). These results were already presented in $[11,15]$.

(ii) Case B: combining intensity mapping and the joint continuum sample [IM $\times$ Cont. (1 sample)]. (iii) Case C: combining intensity mapping and the two separated radio populations [IM $\times$ Cont. (2 samples)].

(iv) Case D: combining the two radio populations [Cont. only (2 samples)].

In all cases we assumed complete overlap between the intensity mapping and continuum surveys (i.e. $f_{\text {sky }}=0.75$ ).

As in the case of LSST, we first look at the feasibility of detecting GR corrections by computing the uncertainty on $\epsilon_{\mathrm{GR}}$ in the most optimistic case, with all parameters, except for $f_{\mathrm{NL}}$, fixed:

$$
\begin{array}{ll}
\sigma_{\mathrm{A}}\left(\epsilon_{\mathrm{GR}}\right)=2.75, & \sigma_{\mathrm{B}}\left(\epsilon_{\mathrm{GR}}\right)=1.40, \\
\sigma_{\mathrm{C}}\left(\epsilon_{\mathrm{GR}}\right)=1.19, & \sigma_{\mathrm{D}}\left(\epsilon_{\mathrm{GR}}\right)=10.4 .
\end{array}
$$

Thus, even in the best-case scenario it would be impossible to observe the effect of the relativistic corrections above $1 \sigma$. Following the argument laid out in Sec. III B it is easy to understand why. First of all, the signal-to-noise level for both types of experiment is significantly lower than for photometric surveys. This is due to the smaller number density of radio sources above $5 \mu \mathrm{Jy}$ as well as the high noise level expected for intensity mapping. Second, unlike in the case of red and blue galaxies, the magnification and evolution biases of the different tracers are not that different from one another.

As was reported in [15], the lack of radial information for continuum surveys makes the constraints on our large-scale observables very sensitive to the priors assumed on any nuisance or cosmological parameter. We can expect that the cross-correlation with intensity mapping should help in breaking some of these degeneracies, and therefore we have studied them in detail. We obtain results that are mostly equivalent to those found for LSST: the constraints on $\epsilon_{\mathrm{GR}}$ and $f_{\mathrm{NL}}$ are insensitive to the prior on the clustering bias, but show a clear dependence on $\Delta f_{\text {evo }}$, although in this case they can be optimized with a looser prior of $\Delta f_{\text {evo }} \sim 1$. The constraints on the amplitude of the lensing magnification term $\epsilon_{\mathrm{WL}}$, on the other hand, are significantly more sensitive to the priors on $b(z)$ and $s(z)$ than in the case of LSST. The main reason for this is that we can only constrain $\epsilon_{\mathrm{WL}}$ through the continuum data, since the lensing term cancels exactly to first order for intensity mapping, and thus the lensing amplitude is more degenerate with other parameters. We show the dependence of $\epsilon_{\mathrm{WL}}$ on $\Delta b$ in Fig. 7.

As we did for LSST, we define an optimistic and pessimistic set of priors, given by $\Delta s=\Delta f_{\text {evo }}=0.1$ and $\Delta s=\Delta f_{\text {evo }}=1$, respectively. In both cases we will assume that the clustering bias can be constrained to a $10 \%$ precision $(\Delta b / b=0.1)$ based on smaller-scale data [60]. 


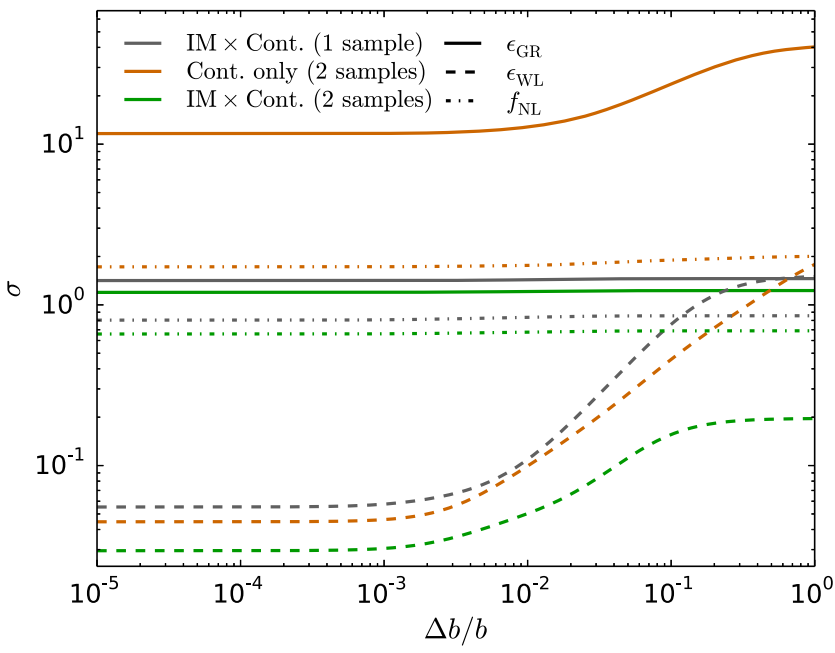

FIG. 7 (color online). Dependence of the constraints on $f_{\mathrm{NL}}$, $\epsilon_{\mathrm{GR}}$ and $\epsilon_{\mathrm{WL}}$ on a constant Gaussian prior on the clustering bias $b(z)$. The constraints on $\epsilon_{\mathrm{WL}}$ exhibit a much stronger dependence on $\Delta b$ due to the fact that lensing magnification can only be constrained through the continuum survey.

Our final constraints on $f_{\mathrm{NL}}$ and $\epsilon_{\mathrm{GR}}$ are shown jointly in Fig. 8 for optimistic priors and listed in Table II. Although the GR corrections remain unobservable for radio experiments, the improvement of the constraints on $f_{\mathrm{NL}}$ due to combining intensity mapping and continuum data is remarkable.

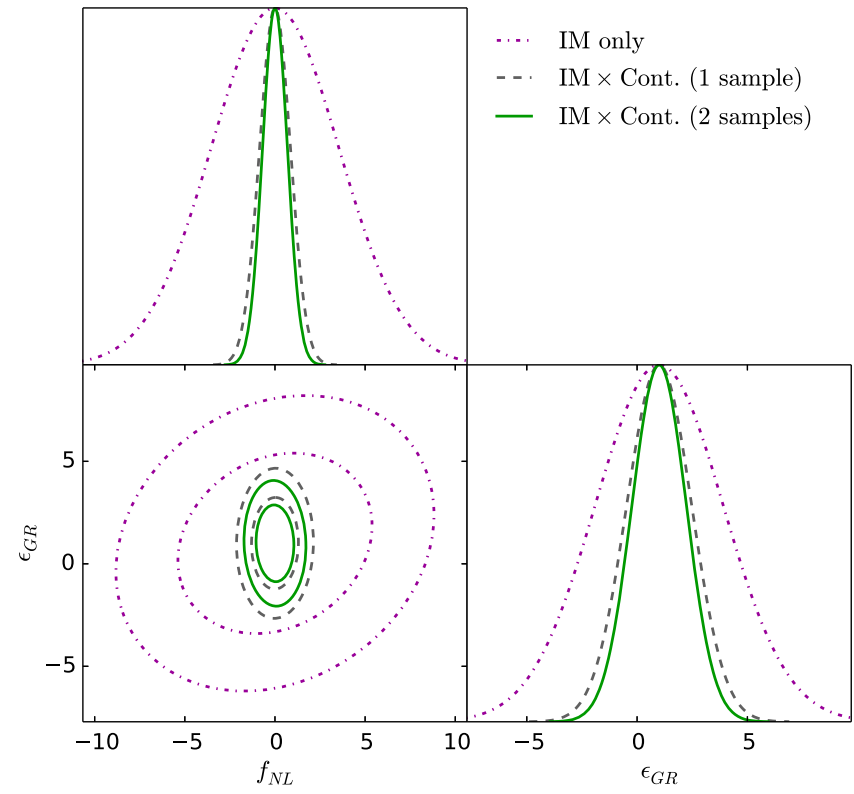

FIG. 8 (color online). Joint constraints for $f_{\mathrm{NL}}$ and $\epsilon_{\mathrm{GR}}$ for the best three combinations of radio experiments: intensity mappingonly (purple), intensity mapping combined with a single continuum sample (grey) and intensity mapping combined with the two different continuum samples (green). Although the GR effects are not detectable, the improvement in the uncertainty on $f_{\mathrm{NL}}$ due to the addition of continuum data is remarkable.

\section{SYNERGIES}

In the last two sections we have described the potential of two key next-generation experiments, SKA-MID and the LSST, to constrain cosmological observables on ultralarge scales, considering the potential multitracer analyses that could be performed using the capabilities of each experiment independently. We would now like to explore the possibility of combining the tracers probed by both surveys jointly, and the potential improvement that this synergy would represent over the results presented above.

Both experiments will be located at similar latitudes and will therefore cover almost equivalent regions of the sky. There should also be a significant redshift overlap between the LSST photometric survey and the SKA-MID HI intensity mapping experiment. The number of potential benefits of cross-correlating the data sets from both experiments is therefore immense, e.g.: the ability of LSST to provide approximate redshifts for many of the sources in the SKA continuum survey, the ability of the SKA to provide more accurate redshift information through its $\mathrm{HI}$ survey to improve the LSST photo- $z$ calibration, or the invaluable cross-checks that will be performed to mitigate the effects of survey-specific systematic uncertainties (see [61] for a recent review of potential synergies). Since such potential benefits cannot be ignored, this synergy will surely take place, and therefore it makes sense to forecast for the potential impact on ultralarge-scale cosmology.

We will present our forecasts in two cases:

(i) Combining SKA-IM and the LSST full sample, without differentiating between red and blue galaxies.

(ii) Combining SKA-IM and the two red and blue LSST samples (i.e. three tracers in total).

We do not consider cross-correlations between LSST and the SKA continuum survey for two reasons. First, a large fraction of the radio galaxies detected by the SKA should be present in the LSST data set too. Therefore these are not completely disjoint tracers, and evaluating their noise cross-power spectrum, crucial for the multitracer method, would be very uncertain. Second, the benefits of combining IM with a photometric survey are clearer: an improved coverage of the small radial scales due to the better spectral resolution of intensity mapping and an improved coverage of small transverse scales due to the better angular resolution of optical surveys. Finally, as we discussed in Sec. VA 1, the low clustering bias, mild evolution bias and the absence of linear-order perturbations in the transverse distance hamper the ability of intensity mapping to constrain $f_{\mathrm{NL}}$ and $\epsilon_{\mathrm{GR}}$ on its own. However, those same characteristics make it a very useful probe to combine with other tracers in the multitracer scheme.

Although both experiments should cover almost the same area of the sky, we can expect a certain loss of overlap caused by regions of the sky dominated by different systematics (e.g. dust extinction for LSST and galactic 


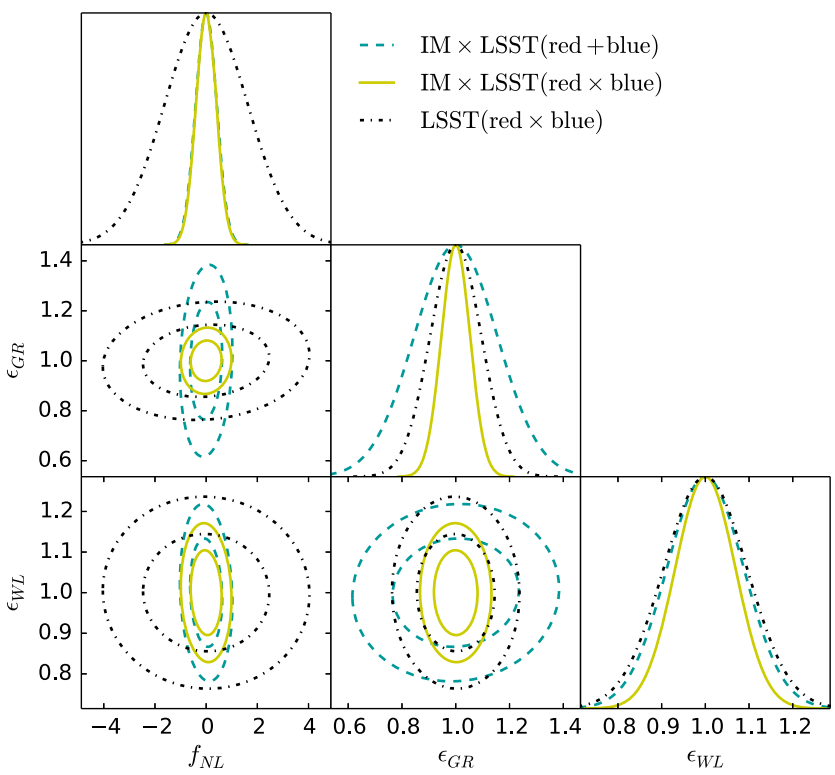

FIG. 9 (color online). Joint constraints on $f_{\mathrm{NL}}, \epsilon_{\mathrm{GR}}$ and $\epsilon_{\mathrm{WL}}$ for the combination of both red and blue galaxies in LSST (black ellipses), for a combination of SKA1-MID intensity mapping with the full LSST sample (cyan) and for intensity mapping combined with both red and blue galaxies for (yellow).

synchrotron for SKA-IM). The forecasts presented here conservatively assume that both experiments will yield useable observations in a patch of area $f_{\text {sky }}=0.4$. Note that we treated this value as the sky fraction in which both experiments can be cross-correlated, however we still use the full SKA area $\left(f_{\text {sky }}=0.75\right)$ when computing the noise power spectrum for intensity mapping.

The full LSST sample was modeled by combining the red and blue luminosity functions, and conservatively assuming a photo- $z$ uncertainty $\sigma_{0}=0.05$. Following the prescription outlined in Sec. IV A, this entails dividing the sample into the same 9 redshift bins used for the blue population.

The joint constraints on $f_{\mathrm{NL}}, \epsilon_{\mathrm{WL}}$ and $\epsilon_{\mathrm{GR}}$ for the two cases above, together with the constraints found for LSST alone are shown in Fig. 9, where an optimistic prior $\Delta s=$ $\Delta f_{\text {evo }}=0.1$ was used. The quantitative results are listed in Table II for both pessimistic and optimistic priors. Several conclusions can be drawn from this exercise:

(i) In spite of the low clustering bias of neutral hydrogen, the errors on $f_{\mathrm{NL}}$ improve immensely by combining LSST and intensity mapping (by a factor of 3-4 compared to the LSST-only case).

(ii) Although we observed that the large evolution bias of red galaxies was crucial in boosting the detection level of GR corrections when jointly analyzing the red and blue samples for LSST, we now see that the cross-correlation of $\mathrm{HI}$ intensity mapping and the full LSST sample, which does not display such a large evolution bias, could still yield a measurement of $\epsilon_{\mathrm{GR}}$ above the $5 \sigma$ level. In the most optimistic case (optimistic priors and a joint analysis of the three samples) this increases to $\sim 20 \sigma$.

(iii) Due to the cancellation of the lensing term for intensity mapping the improvement in the detection of the large-scale lensing magnification is only mild.

\section{DISCUSSION}

In this paper we have shown how more than one tracer of the cosmic density field can be used to extract precise measurements of the ultralarge-scale features of the universe. We have shown that a judicious combination of photometric redshift surveys with cosmological radio surveys will allow us to obtain precise constraints of primordial non-Gaussianity, of weak lensing on large scales and most significantly, of general-relativistic effects which depend on various combinations of the gravitational potential. Our final results are summarized in Table II in terms of the marginalized uncertainties on $f_{\mathrm{NL}}$ and the amplitudes of the GR corrections $\left(\epsilon_{\mathrm{GR}}\right)$ and lensing magnification $\left(\epsilon_{\mathrm{WL}}\right)$.

More specifically, we have shown that the best way to optimize the detection of GR effects is to use a combination of low-noise tracers (i.e. high number density) with very different evolution and magnification biases. We find that ongoing and future photometric redshift surveys will be able to satisfy both conditions, given the large expected number density of observed sources and the possibility of separating the galaxy sample into two populations, blue and red galaxies, with different evolution of their luminosity functions. Indeed we have seen how the rapid decay in the number density of red galaxies above redshift $\sim 1$ translates itself into a large $f_{\text {evo }}$ that boosts the signal of the GR corrections and could enable LSST to detect them with a significance of $\sim 5-10 \sigma$. Using the same method we have also shown that DES might be able to make a lower significance detection $(\sim 3 \sigma)$ in a shorter term.

We have also explored the possibility of combining two of the main cosmological radio probes, HI intensity mapping and continuum surveys, planned for the Square Kilometre Array. We have seen that in this case, the higher noise levels make a detection of the GR corrections unviable, in spite of the larger volume covered in comparison with LSST. However, the contrast between the low clustering bias of neutral hydrogen and its large value for certain radio sources makes the combined analysis of both probes very beneficial to reduce the uncertainties on the level of primordial non-Gaussianity. We have seen that, in the worst-case scenario (1 single continuum sample and pessimistic priors), the error on $f_{\mathrm{NL}}$ could improve by a factor of 3 with respect to the constraints from intensity mapping alone.

Finally, since LSST and SKA will carry out their observations over a very similar region of the sky, the benefits of cross-correlating both experiments will be immense for many science cases, and here we have studied 


\section{ALONSO AND P. G. FERREIRA}

the potential impact of this synergy in the study of cosmology on ultralarge scales. We have shown that it would be possible to improve the constraints on $f_{\mathrm{NL}}$ by a factor of at least 2-3 with respect to the individual constraints from each experiment. The detection level of the GR effects would also improve significantly and, more interestingly, the combination of the full LSST sample and SKA1 intensity mapping should also be able to detect these effects above $5 \sigma$ regardless of the large evolution bias of red galaxies.

We have shown that the detectability of these new effects is an exciting prospect, yet many important challenges must be overcome before such measurements can be carried out. First, the estimates of clustering statistics on ultralarge scales in most experiments are riddled with systematic uncertainties. Intensity mapping is a perfect example of this: galactic and extragalactic foregrounds have radio intensities that are several orders of magnitude larger than the cosmological HI signal. Although both components can in principle be separated based on their very different spectral behavior, any foreground residual will necessarily contaminate the signal on large scales, both angular (due to the large-scale features of galactic foregrounds) and radial (due to their smooth frequency dependence). As shown in [62], imperfect foreground cleaning can lead to the crosscorrelations between well-separated frequency bins being unreliable, and, as was reported in [15], excluding these cross-correlations from the analysis can have a very detrimental effect on the uncertainty on large-scale parameters such as $f_{\mathrm{NL}}$. Other instrumental issues, such as the effect of correlated $1 / f$ noise in the data, a bad characterization of the instrument's beam or the contamination from polarized foregrounds due to polarization leakage can introduce even more troublesome systematics in the data that must be correctly minimized in order to make these measurements possible.

Optical surveys are also subject to a sizeable collection of systematics. Effects such as galactic dust extinction and air mass can modify the observed magnitude and generate a nonuniform magnitude limited sample which can modify the observed clustering statistics on large scales. Star contamination, i.e. the accidental inclusion of stars in the galaxy sample, and star obscuration, as reported in [63], can also have a significant impact on the clustering observed on large angular distances, since both effects grow toward the galactic plane. Another specific source of systematic uncertainty for photometric surveys is the incorrect characterization of the window functions corresponding to each redshift bin. This can be caused by uncertainties in the galaxy redshift distribution, due to the absence of precise redshifts, and to inaccuracies in the characterization of the photo- $z$ distribution as a function of redshift.

Most of these systematic effects, unless corrected for, will limit our ability to use the observations on the largest
PHYSICAL REVIEW D 92, 063525 (2015)

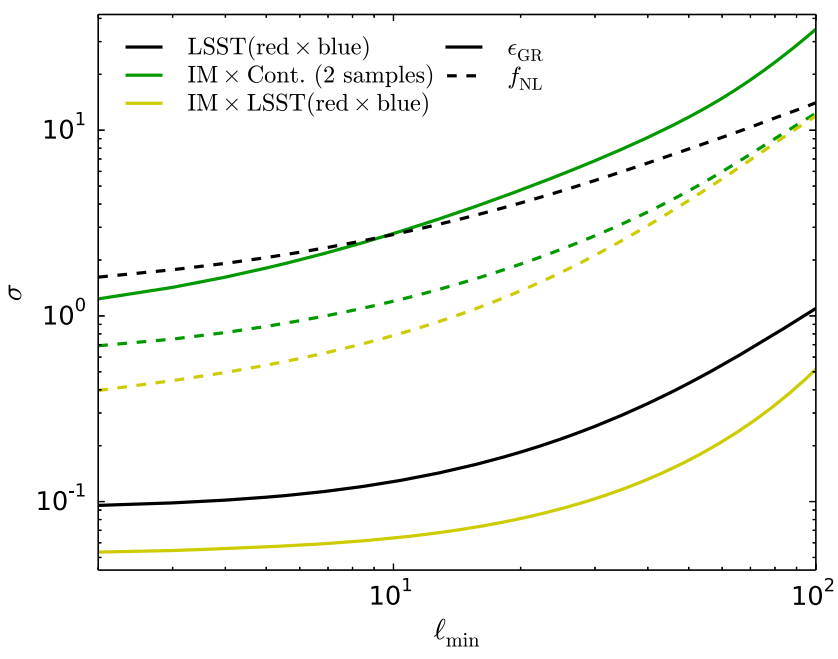

FIG. 10 (color online). Dependence of the constraints on $\epsilon_{\mathrm{GR}}$ (solid) and $f_{\mathrm{NL}}$ (dashed) on the largest angular scale included in the analysis (given by the minimum multipole $\ell_{\min }$ ), for three different tracer combinations: LSST red and blue galaxies (black), intensity mapping and our 2 continuum samples (green) and IM combined with both LSST samples (yellow). The constraints degrade significantly after $\ell_{\min } \sim 10$.

angular scales. We have studied the dependence of our forecasts on the largest scale included in the analysis (i.e. the minimum angular multipole $\ell_{\min }$ ) in Fig. 10 for our best three tracer combinations (LSST red and blue, IM combined with the two continuum samples and IM combined with the two LSST samples). The constraints on $f_{\mathrm{NL}}$ and $\epsilon_{\mathrm{GR}}$ degrade significantly above $\ell_{\text {min }} \sim 10$.

In addition to these systematic effects, we have also identified other challenges that must be tackled before this detection can be achieved. We have found that having at least two tracers with very different magnification and/or evolution biases is key to boosting the signal of GR effects, and we have illustrated this point with the case of red and blue galaxies in photometric surveys. However, we have also seen that, even if such tracers can be found, a sufficiently precise knowledge of their joint magnitude-redshift distribution is necessary to statistically detect the GR effects, which would otherwise be swamped by the uncertainties on $s(z)$ and $f_{\text {evo. }}$. In the case of LSST we have shown that priors of the order of $\Delta s \simeq \Delta f_{\text {evo }} \simeq 0.1$ would be needed to optimize this measurement. To put these uncertainties into perspective, we should note that the uncertainty in the value of $f_{\text {evo }}$ propagated from the errors in the estimate of the luminosity function for red galaxies made by [42], based on data from the DEEP2 and COMBO-17 surveys, is of the order of $\Delta f_{\text {evo }} \sim 2$ at high redshifts $(z \gtrsim 0.8)$, where the GR effects are maximally boosted. Assuming that this uncertainty decreases with the square-root of the observed number of galaxies, spectroscopic observations of a representative sample of $\sim 1.5$ million galaxies with 
redshifts above $\sim 0.8$ would be needed to achieve the optimal sensitivity. This would correspond to a fraction of $\sim 2 \%$ of the total red sample expected for LSST in the same redshift range. The Dark Energy Spectroscopic Instrument (DESI) [64], for instance, should be able to measure spectra for $\sim 4$ million luminous red galaxies in the redshift range $0.4 \lesssim z \lesssim 1.0$, although these observations would be carried out in the northern hemisphere.

Finally, it is worth noting that the detection levels and uncertainties reported here depend critically on the fiducial models used for the bias functions of the tracers under study. Although we have tried to use reliable and conservative assumptions for these models, these quantities are still uncertain, and therefore the quantitative results could change as those uncertainties are reduced. On the other hand, this also implies that these results could actually be improved if one could find more optimal tracer classifications (e.g. beyond the traditional red-blue split) enhancing the amplitude of the relativistic corrections.

If we can tackle all these challenges, then, by measuring ultralarge-scale modes in the density field, we will be able to break out of what has been the conventional arena of large scale structure studies - the quasistatic regime-on substantially subhorizon scales. Until now, most surveys of large-scale structure (such as redshift or weak lensing surveys) have probed what can be thought of as the Newtonian regime of cosmological perturbation theory, where the time dependence of the gravitational potentials can be ignored [65]. Now, by probing scales which span the cosmological horizon, this time dependence comes into play, giving us a completely new window on the evolution of cosmic perturbations.

Accessing ultralarge scales can be of particular significance in constraining modifications to general relativity [27]. It has been shown that in the quasistatic regime, cosmological data will only constrain a limited subspace of possible modifications to gravity [66-68]. To extend the scope of the constraints, it is essential to break any degeneracies that arise on small scales by using the time variation of the gravitational potentials on large scales.

In order to study the constraining power of the relativistic terms explored in this work it would then be desirable to connect their amplitude with specific modified gravity theories. Work in this direction is already underway and, as shown by $[69,70]$ one should expect specific signatures of the order of a few percent in the amplitude of the largescale power spectrum. The detectability of these modifications, as well as the connection with particular models is still not clear, and we intend to pursue this line of investigation in future work.

Given that we are targeting ultralarge scales, one should bear in mind the mild evidence for deviations from the standard, $\Lambda \mathrm{CDM}$, concordance model on large scales. The recent Planck measurements of the $\mathrm{CMB}$ on large scales has found a number of anomalies-suppression of power on very large angles, correlations between the large angle multipoles and an anomalously cold spot—which have yet to be understood [71]. Cosmic variance severely restricts any attempts at better characterizing these anomalies with the CMB alone. One would hope, however, that with the possibility of better constraining large scale modes with multiple tracers, one could substantially improve our characterization of these large scale anomalies with complementary constraints.

We have focused on extracting information at the level of auto and cross-correlations of the various tracers. An interesting, uncharted, avenue would be to attempt to use these techniques to extract maps of each of the effects that come into play. In other words, and much like what is done in the case of the CMB [72], one could imagine constructing linear combinations of the various observables (which are three dimensional maps of a particular tracer) to extract, for example, maps of the gravitational potential, their derivatives, large scale flows, etc. This is a somewhat ambitious goal but if achievable would open up a completely new window into the large scale properties of space-time and matter; a direct comparison between the different maps, and a comparison between these maps and other cosmic observables might be used to uncover different cosmological properties which are usually buried in the standard correlation function (or power spectrum) analysis.

Finally, we have focused on a particular choice of current and future surveys-photometric redshift surveys and cosmological radio surveys-but this does not cover all possible observational approaches. Spectroscopic galaxy redshift surveys, such as Euclid [73] and WFIRST [74], will also attempt to constrain the effects we discuss in this paper, albeit on smaller scales and with a lower source density. A more speculative, ambitious and, in our mind, exciting approach using an all sky, wide band survey, SPHEREX, would have all the right characteristics for the methods proposed here. As argued in [75], the number density and bias of these sources should be sufficiently large to aid, and complement, the constraints that will be found with the combination of probes found here. In addition to these probes of galaxy clustering, it would also be worth exploring the potential benefits of combining them with other cosmological observables, such as weak lensing, CMB lensing or even the CMB itself. These combinations could benefit from the particular properties of these tracers (e.g. zero bias for weak lensing and minimal clustering variance for the $\mathrm{CMB}$ ), significantly improving the constraints reported here. We are clearly entering an era in which precision measurement of ultralarge-scale modes can become a core science goal of observational cosmology. 


\section{ACKNOWLEDGMENTS}

We thank Phil Bull, Elisa Chisari, Jo Dunkley, Ruth Durrer, Roy Maartens, Francesco Montanari and Mario Santos for useful comments and discussions. D. A. is supported by ERC Grant No. 259505. P. G. F. acknowledges support from STFC, BIPAC and the Oxford Martin School.

\section{APPENDIX: THE LINEAR ORDER COMPONENTS OF THE FLUCTUATION IN SOURCE NUMBER COUNTS}

The complete expressions, to linear order, for the transfer function of the perturbation in the source number counts used in Eq. (9) is given by [20]:

$$
\begin{gathered}
\Delta_{\ell}^{\mathrm{D}, i}(k) \equiv \int d \eta b \tilde{W}_{i} \delta_{M, \mathrm{syn}}(k, \eta) j_{\ell}(k \chi(\eta)), \quad \Delta_{\ell}^{\mathrm{RSD}, i}(k) \equiv \int d \eta(a H)^{-1} \tilde{W}_{i}(\eta) \theta(k, \eta) j_{\ell}^{\prime \prime}(k \chi(\eta)), \\
\Delta_{\ell}^{\mathrm{L}, i}(k) \equiv \ell(\ell+1) \int d \eta \tilde{W}_{i}^{\mathrm{L}}(\eta)(\phi+\psi)(k, \eta) j_{\ell}(k \chi(\eta)), \quad \Delta_{\ell}^{\mathrm{V} 1, i}(k) \equiv \int d \eta\left(f_{\mathrm{evo}}-3\right) a H \tilde{W}_{i}(\eta) \frac{\theta(k, \eta)}{k^{2}} j_{\ell}(k \chi(\eta)), \\
\Delta_{\ell}^{\mathrm{V} 2, i}(k) \equiv \int d \eta\left(1+\frac{H^{\prime}}{a H^{2}}+\frac{2-5 s}{\chi a H}+5 s-f_{\mathrm{evo}}\right) \tilde{W}_{i}(\eta) \frac{\theta(k, \eta)}{k} j_{\ell}^{\prime}(k \chi(\eta)), \\
\Delta_{\ell}^{\mathrm{P} 1, i}(k) \equiv \int d \eta\left(2+\frac{H^{\prime}}{a H^{2}}+\frac{2-5 s}{\chi a H}+5 s-f_{\mathrm{evo}}\right) \tilde{W}_{i}(\eta) \psi(k, \eta) j_{\ell}(k \chi(\eta)), \\
\Delta_{\ell}^{\mathrm{P} 2, i}(k) \equiv \int d \eta(5 s-2) \tilde{W}_{i}(\eta) \phi(k, \eta) j_{\ell}(k \chi(\eta)), \quad \Delta_{\ell}^{\mathrm{P} 3, i}(k) \equiv \int d \eta(a H)^{-1} \tilde{W}_{i}(\eta) \phi^{\prime}(k, \eta) j_{\ell}(k \chi(\eta)), \\
\Delta_{\ell}^{\mathrm{P} 4, i}(k) \equiv \int d \eta \tilde{W}_{i}^{\mathrm{P} 4}(\eta)(\phi+\psi)(k, \eta) j_{\ell}(k \chi(\eta)), \quad \Delta_{\ell}^{\mathrm{ISW}, i}(k) \equiv \int d \eta \tilde{W}_{i}^{\mathrm{ISW}}(\eta)(\phi+\psi)^{\prime}(k, \eta) j_{\ell}(k \chi(\eta)),
\end{gathered}
$$

where $j_{\ell}(x)$ is the spherical Bessel function of order $\ell$ and where we have defined the window functions

$$
\begin{aligned}
\tilde{W}_{i}(\eta(z)) & \equiv W_{i}(z)\left(\frac{d \eta}{d z}\right)^{-1}, \quad \tilde{W}_{i}^{\mathrm{L}}(\eta) \equiv \int_{0}^{\eta} d \eta^{\prime} \tilde{W}_{i}\left(\eta^{\prime}\right) \frac{2-5 s\left(\eta^{\prime}\right)}{2} \frac{\chi(\eta)-\chi\left(\eta^{\prime}\right)}{\chi(\eta) \chi\left(\eta^{\prime}\right)}, \\
\tilde{W}_{i}^{\mathrm{P} 4}(\eta) & \equiv \int_{0}^{\eta} d \eta^{\prime} \tilde{W}_{i}\left(\eta^{\prime}\right) \frac{2-5 s}{\chi}, \quad \tilde{W}_{i}^{\mathrm{ISW}}(\eta) \equiv \int_{0}^{\eta} d \eta^{\prime} \tilde{W}_{i}\left(\eta^{\prime}\right)\left(1+\frac{H^{\prime}}{a H^{2}}+\frac{2-5 s}{\chi a H}+5 s-f_{\text {evo }}\right) .
\end{aligned}
$$

The quantities $\delta_{M \text {,syn }}, \theta, \phi$ and $\psi$ in the equations above are, respectively: the transfer function for the matter density perturbations in the comoving synchronous gauge, the transfer function for the peculiar velocity divergence in the conformal Newtonian gauge and transfer functions for the two metric potentials in the same gauge, defined by the line element:

$$
d s^{2}=-a^{2}(\eta)\left[(1+2 \psi) d \eta^{2}-(1-2 \phi) \delta_{i j} d x^{i} d x^{j}\right] .
$$

[1] C. Bonvin, R. Durrer, and M. A. Gasparini, Phys. Rev. D 73, 023523 (2006).

[2] J. Yoo, A. L. Fitzpatrick, and M. Zaldarriaga, Phys. Rev. D 80, 083514 (2009).

[3] J. Yoo, Phys. Rev. D 82, 083508 (2010).

[4] C. Bonvin and R. Durrer, Phys. Rev. D 84, 063505 (2011).
[5] A. Challinor and A. Lewis, Phys. Rev. D 84, 043516 (2011).

[6] N. Dalal, O. Doré, D. Huterer, and A. Shirokov, Phys. Rev. D 77, 123514 (2008).

[7] S. Matarrese and L. Verde, Astrophys. J. 677, L77 (2008). 
[8] T. Giannantonio, A. J. Ross, W. J. Percival, R. Crittenden, D. Bacher, M. Kilbinger, R. Nichol, and J. Weller, Phys. Rev. D 89, 023511 (2014).

[9] T. Namikawa, T. Okamura, and A. Taruya, Phys. Rev. D 83, 123514 (2011).

[10] T. Giannantonio, C. Porciani, J. Carron, A. Amara, and A. Pillepich, Mon. Not. R. Astron. Soc. 422, 2854 (2012).

[11] S. Camera, M. G. Santos, P. G. Ferreira, and L. Ferramacho, Phys. Rev. Lett. 111, 171302 (2013).

[12] L. D. Ferramacho, M. G. Santos, M. J. Jarvis, and S. Camera, Mon. Not. R. Astron. Soc. 442, 2511 (2014).

[13] S. Camera, M. G. Santos, and R. Maartens, Mon. Not. R. Astron. Soc. 448, 1035 (2015).

[14] A. Raccanelli, O. Doré, D. J. Bacon, R. Maartens, M. G. Santos, S. Camera, T. M. Davis, M. J. Drinkwater, M. Jarvis, R. Norris et al., J. Cosmol. Astropart. Phys. 01 (2015) 042.

[15] D. Alonso, P. Bull, P. G. Ferreira, R. Maartens, and M. G. Santos, arXiv:1505.07596.

[16] U. Seljak, Phys. Rev. Lett. 102, 021302 (2009).

[17] L. R. Abramo and K. E. Leonard, Mon. Not. R. Astron. Soc. 432, 318 (2013).

[18] M. Bruni, R. Crittenden, K. Koyama, R. Maartens, C. Pitrou, and D. Wands, Phys. Rev. D 85, 041301 (2012).

[19] T. Baldauf, U. Seljak, L. Senatore, and M. Zaldarriaga, J. Cosmol. Astropart. Phys. 10 (2011) 031.

[20] E. Di Dio, F. Montanari, J. Lesgourgues, and R. Durrer, J. Cosmol. Astropart. Phys. 11 (2013) 044.

[21] T. Matsubara, Astrophys. J. Lett. 537, L77 (2000).

[22] N. Kaiser, Mon. Not. R. Astron. Soc. 227, 1 (1987).

[23] I. I. Shapiro, Phys. Rev. Lett. 13, 789 (1964).

[24] R. K. Sachs and A. M. Wolfe, Astrophys. J. 147, 73 (1967).

[25] R. Scranton, B. Ménard, G. T. Richards, R. C. Nichol, A. D. Myers, B. Jain, A. Gray, M. Bartelmann, R. J. Brunner, A. J. Connolly et al., Astrophys. J. 633, 589 (2005).

[26] H. Hildebrandt, L. van Waerbeke, and T. Erben, Astron. Astrophys. 507, 683 (2009).

[27] A. Hall, C. Bonvin, and A. Challinor, Phys. Rev. D 87, 064026 (2013).

[28] D. Baumann, arXiv:0907.5424.

[29] E. Komatsu and D. N. Spergel, Phys. Rev. D 63, 063002 (2001).

[30] Planck Collaboration, arXiv:1502.01592.

[31] D. Jeong, F. Schmidt, and C. M. Hirata, Phys. Rev. D 85, 023504 (2012).

[32] D. Bertacca, R. Maartens, A. Raccanelli, and C. Clarkson, J. Cosmol. Astropart. Phys. 10 (2012) 025.

[33] J. Lesgourgues, arXiv:1104.2932.

[34] This code is made publicly available at http:// intensitymapping.physics.ox.ac.uk/codes.html.

[35] Planck Collaboration, Astron. Astrophys. 571, A16 (2014).

[36] F. Beutler, C. Blake, M. Colless, D. H. Jones, L. StaveleySmith, L. Campbell, Q. Parker, W. Saunders, and F. Watson, Mon. Not. R. Astron. Soc. 416, 3017 (2011).

[37] C. Blake, T. Davis, G. B. Poole, D. Parkinson, S. Brough, M. Colless, C. Contreras, W. Couch, S. Croom, M. J. Drinkwater et al., Mon. Not. R. Astron. Soc. 415, 2892 (2011).

[38] C. P. Ahn, R. Alexandroff, C. A. Prieto, S. F. Anderson, T. Anderton, B. H. Andrews, É. Aubourg, S. Bailey,
E. Balbinot, R. Barnes et al., Astrophys. J. Suppl. Ser. 203, 21 (2012).

[39] P. A. Abell, J. Allison, S. F. Anderson, J. R. Andrew, J. R. P. Angel, L. Armus, D. Arnett, S. J. Asztalos, T. S. Axelrod et al. (LSST Collaboration), arXiv:0912.0201.

[40] T. Abbot (The Dark Energy Survey Collaboration), arXiv: astro-ph/0510346.

[41] I. Strateva, Ž. Ivezić, G. R. Knapp, V. K. Narayanan, M. A. Strauss, J. E. Gunn, R. H. Lupton, D. Schlegel, N. A. Bahcall, J. Brinkmann et al., Astron. J. 122, 1861 (2001).

[42] S. M. Faber, C. N. A. Willmer, C. Wolf, D. C. Koo, B. J. Weiner, J. A. Newman, M. Im, A. L. Coil, C. Conroy, M. C. Cooper et al., Astrophys. J. 665, 265 (2007).

[43] A. Gabasch, U. Hopp, G. Feulner, R. Bender, S. Seitz, R. P. Saglia, J. Snigula, N. Drory, I. Appenzeller, J. Heidt et al., Astron. Astrophys. 448, 101 (2006).

[44] D. H. Weinberg, R. Davé, N. Katz, and L. Hernquist, Astrophys. J. 601, 1 (2004).

[45] A. L. Coil, J. A. Newman, D. Croton, M. C. Cooper, M. Davis, S. M. Faber, B.F. Gerke, D. C. Koo, N. Padmanabhan, R. H. Wechsler et al., Astrophys. J. 672, 153 (2008).

[46] O. Ilbert, S. Arnouts, H. J. McCracken, M. Bolzonella, E. Bertin, O. Le Fèvre, Y. Mellier, G. Zamorani, R. Pellò, A. Iovino et al., Astron. Astrophys. 457, 841 (2006).

[47] F. Montanari and R. Durrer, arXiv:1506.01369.

[48] P. E. Dewdney, P. J. Hall, R. T. Schilizzi, and T. J. L. W. Lazio, The Square Kilometre Array (IEEE, New York, 2009), Vol. 97, p. 1482.

[49] R. Braun, T. L. Bourke, J. G. Green, E. F. Keane, and J. Wagg, Proc. Sci., AASKA14 (2015) 174.

[50] K. Bandura, G. E. Addison, M. Amiri, J. R. Bond, D. Campbell-Wilson, L. Connor, J.-F. Cliche, G. Davis, M. Deng, N. Denman et al., Proc. SPIE Int. Soc. Opt. Eng. 9145, 914522 (2014).

[51] R. A. Battye, M. L. Brown, I. W. A. Browne, R. J. Davis, P. Dewdney, C. Dickinson, G. Heron, B. Maffei, A. Pourtsidou, and P. N. Wilkinson, arXiv:1209.1041.

[52] R. A. Battye, R. D. Davies, and J. Weller, Mon. Not. R. Astron. Soc. 355, 1339 (2004).

[53] J. S. B. Wyithe and A. Loeb, Mon. Not. R. Astron. Soc. 383, 606 (2008).

[54] T.-C. Chang, U.-L. Pen, J. B. Peterson, and P. McDonald, Phys. Rev. Lett. 100, 091303 (2008).

[55] P. Bull, P. G. Ferreira, P. Patel, and M. G. Santos, Astrophys. J. 803, 21 (2015).

[56] M. S. Yun, N. A. Reddy, and J. J. Condon, Astrophys. J. 554, 803 (2001).

[57] Y. Ueda, M. Akiyama, K. Ohta, and T. Miyaji, Astrophys. J. 598, 886 (2003).

[58] C. J. Willott, S. Rawlings, K. M. Blundell, M. Lacy, and S. A. Eales, Mon. Not. R. Astron. Soc. 322, 536 (2001).

[59] R. J. Wilman, L. Miller, M. J. Jarvis, T. Mauch, F. Levrier, F. B. Abdalla, S. Rawlings, H.-R. Klöckner, D. Obreschkow, D. Olteanu et al., Mon. Not. R. Astron. Soc. 388, 1335 (2008).

[60] S. N. Lindsay, M. J. Jarvis, and K. McAlpine, Mon. Not. R. Astron. Soc. 440, 2322 (2014). 
[61] D. Bacon, S. Bridle, F. B. Abdalla, M. Brown, P. Bull, S. Camera, R. Fender, K. Grainge, Z. Ivezic, M. Jarvis et al., Proc. Sci., AASKA14 (2015) 145 [arXiv:1501.03977].

[62] D. Alonso, P. Bull, P. G. Ferreira, and M. G. Santos, Mon. Not. R. Astron. Soc. 447, 400 (2015).

[63] A. J. Ross, S. Ho, A. J. Cuesta, R. Tojeiro, W. J. Percival et al., Mon. Not. R. Astron. Soc. 417, 1350 (2011).

[64] M. Levi, C. Bebek, T. Beers, R. Blum, R. Cahn, D. Eisenstein, B. Flaugher, K. Honscheid, R. Kron, O. Lahav et al., arXiv:1308.0847.

[65] J. Noller, F. von Braun-Bates, and P. G. Ferreira, Phys. Rev. D 89, 023521 (2014).

[66] T. Baker, P. G. Ferreira, and C. Skordis, Phys. Rev. D 89, 024026 (2014).
[67] T. Baker, P. G. Ferreira, C. D. Leonard, and M. Motta, Phys. Rev. D 90, 124030 (2014).

[68] C. D. Leonard, T. Baker, and P. G. Ferreira, Phys. Rev. D 91, 083504 (2015).

[69] L. Lombriser and A. Taylor, arXiv:1505.05915.

[70] T. Baker and P. Bull, arXiv:1506.00641.

[71] P. Ade et al. (Planck), arXiv:1506.07135.

[72] R. G. Crittenden and N. Turok, Phys. Rev. Lett. 76, 575 (1996).

[73] R. Laureijs et al. (EUCLID), arXiv:1110.3193.

[74] J. Green, P. Schechter, C. Baltay, R. Bean, D. Bennett et al., arXiv:1208.4012.

[75] O. Dore, J. Bock, P. Capak, R. de Putter, T. Eifler et al., arXiv:1412.4872. 